\title{
Isotropic-nematic spinodal decomposition kinetics
}

\author{
Jan K. G. Dhont ${ }^{1}$ and W. J. Briels ${ }^{2}$ \\ ${ }^{1}$ Forschungszentrum Jülich, Institut für Festkörper Forschung (IFF), Weiche Materie, D-52425 Jülich, Germany \\ ${ }^{2}$ Computational Dispersion Rheology, Department of Applied Physics, University of Twente, Postbus 217, \\ 7500 AE Enschede, The Netherlands
}

(Received 22 April 2005; published 13 September 2005)

\begin{abstract}
The initial stage of isotropic-nematic spinodal demixing kinetics of suspensions of very long and thin, stiff, repulsive rods is analyzed on the basis of the $N$-particle Smoluchowski equation. Equations of motion for the reduced probability density function of the position and orientation of a rod are expanded up to second order in spatial gradients and leading order in orientational order parameter. The resulting equation of motion is solved analytically, from which the temporal evolution of light-scattering patterns are calculated. It is shown that inhomogeneities in number density are enslaved by the temporal development of inhomogeneities in orientational order. Furthermore, demixing due to rotational diffusion is shown to be much faster as compared to translational diffusion. This results in an instable mode that is rotational, for which the corresponding eigenvector remains finite at zero wave vector. The scattered intensity nevertheless exhibits a maximum at a finite wave vector due to the wave-vector dependence of time-exponential prefactors. The wave vector where the intensity exhibits a maximum is therefore predicted to be a function of time even during the initial stage of demixing.
\end{abstract}

DOI: 10.1103/PhysRevE.72.031404

PACS number(s): 82.70.-y, 64.75.+g, 61.30.-v

\section{INTRODUCTION}

The initial stage of spinodal gas-liquid demixing kinetics of simple molecular mixtures and suspensions of spherical colloidal particles has been described by Cahn and Hilliard $[1,2]$, on the basis of irreversible thermodynamics. Extensions of the Cahn-Hilliard theory based on various types of equations of motion have been proposed (see, for example, Refs. [3-11]) where in some cases attempts are made to describe later stages of gas-liquid demixing. In addition, a microscopic approach based on the $N$-particle Smoluchowski equation for suspensions of spherical colloids has been developed which includes both the initial and intermediate stages of demixing [12-14]. For the later stages of demixing, where relatively large density differences and sharp interfaces exist, scaling arguments exist for the temporal development of inhomogeneities (see, for example, Refs. [15,16]). Many experiments on spinodal demixing of simple molecular mixtures have been reported (overviews can be found in Refs. [17-23], where additional related references can be found as well), while recently confocal microscopy experiments have been performed on a suspension of spherical colloids [24].

Contrary to the above mentioned work on the gas-liquid phase transition, there are very few theories and hardly any systematic experimental work on the kinetics of isotropicnematic demixing of rodlike systems.

In two important theoretical papers [25,26], demixing kinetics is analyzed with the neglect of rotational diffusion. As a result of the neglect of rotational motion, the unstable eigenmode is purely translational and the corresponding eigenvalue therefore varies like $\sim k^{2}$ (where $k$ is the wave vector). In the present paper we investigate the rotational contribution to demixing kinetics. As will be shown, rotational diffusion is the dominant mechanism for isotropic-nematic spinodal decomposition kinetics of repulsive rods.The present analysis predicts that the unstable eigenmode is predominantly rotational, so that the corresponding eigenvalue remains finite at zero wave vector. As a result, the scattering wave vector where the scattered intensity exhibits a maximum is time dependent, even during the initial stage of demixing.

The only systematic experimental study on isotropicnematic phase-transition kinetics of suspensions we are aware of is on fd-virus suspensions, where the transition is induced by an external magnetic field [27]. The fd concentration is chosen just below the isotropic-nematic binodal without an external field. On applying a magnetic field, the binodal shifts to lower concentrations. This enables to induce an isotropic-nematic transition by applying a magnetic field. The phase separation kinetics is then probed in time, after switching on the magnetic field, by means of birefringence measurements. A visualization of the temporal development of nematic ordering from simulations can be found in Ref. [28]. This study provides qualitative insight, based on visual analysis of the ordering process, where the kinetics of the nonconserved order parameter has not been investigated quantitatively. It would be worthwhile to infer from these simulations whether the unstable eigenmode is predominantly translational or rotational.

In the present paper we will first derive the relevant equation of motion from the $N$-particle Smoluchowski equation. This equation of motion has been put forward before in Refs. [29,30], and is used in Refs. [25,26] to describe spinodal demixing. The present microscopic approach reveals the approximations under which this equation of motion is valid. The deviation of the probability density function from the initially homogeneous and isotropic state will be assumed small, so that the equation of motion may be linearized with respect to this deviation. During the initial stage of demixing, gradients in inhomogeneities as well as the orientational order parameter are small. This allows us to expand the equa- 
tion of motion with respect to gradients in inhomogeneities and the orientational order parameter. The expansion with respect orientational order is done in terms of orthogonal polyadic products of the unit vector that specifies the orientation of a rod (rather than using spherical harmonics), which allows for an explicit analytic solution of the equation of motion. This solution is used to calculate scattering patterns from demixing solutions of rods that can be compared to experiments. When demixing is dominated by translational diffusion, the scattering pattern in the initial stage exhibits a maximum as a function of the wave vector which grows in intensity at a constant wave vector, independent of time. When demixing is dominated by rotational diffusion, the scattering pattern also exhibits a ring shape, but the wave vector where the intensity exhibits its maximum shifts to lower values, even during the initial stage of demixing. Formally, this is due to the wave-vector dependence of the unstable eigenvalue of the relevant equation(s) of motion. This eigenvalue exhibits a minimum in its wave-vector dependence and is zero at zero wave vector when translational diffusion dominates. When rotational diffusion dominates, the eigenvalue is monotonically increasing with increasing wave vector and is nonzero at zero wave vector. It is discussed how scattering experiments can be used to verify or falsify theories on isotropic-nematic spinodal demixing.

\section{EQUATION OF MOTION}

The fundamental equation of motion is derived in this section, on the basis of which demixing kinetics will be discussed starting from an isotropic state.

The equation of motion for the probability density function (pdf) $P(\mathbf{r}, \hat{\mathbf{u}}, t)$ for the position $\mathbf{r}$ and orientation $\hat{\mathbf{u}}$ of a rod can be obtained from the $N$-particle Smoluchowski equation. This is the overdamped equation of motion for the pdf of the positions $\mathbf{r}_{j}$ and orientations $\hat{\mathbf{u}}_{j}$ with $j=1,2, \ldots N$ of the $n$ rods in the system under consideration. Neglecting hydrodynamic interactions between the colloidal rods this equation reads $[31,32]$

$$
\begin{aligned}
\frac{\partial P}{\partial t}= & \sum_{j=1}^{N}\left\{D_{t} \boldsymbol{\nabla}_{j} \cdot \mathbf{D}\left(\hat{\mathbf{u}}_{j}\right) \cdot\left[\boldsymbol{\nabla}_{j} P+\beta P \boldsymbol{\nabla}_{j} \Phi\right]\right. \\
& \left.+D_{r} \hat{\mathcal{R}}_{j} \cdot\left[\hat{\mathcal{R}}_{j} P+\beta P \hat{\mathcal{R}}_{j} \Phi\right]\right\},
\end{aligned}
$$

with $\boldsymbol{\nabla}_{j}$ the gradient operator with respect to $\mathbf{r}_{j}$ and,

$$
\hat{\mathcal{R}}_{j}(\cdots)=\hat{\mathbf{u}}_{j} \times \nabla_{\hat{\mathbf{u}}_{j}}(\cdots),
$$

the rotational operator with respect to $\hat{\mathbf{u}}_{j}$, where $\boldsymbol{\nabla}_{\hat{\mathbf{u}}_{j}}$ is the gradient operator with respect to the Cartesian coordinates of $\hat{\mathbf{u}}_{j}$. Furthermore,

$$
\mathbf{D}(\hat{\mathbf{u}})=[\hat{\mathbf{I}}+\hat{\mathbf{u}} \hat{\mathbf{u}}], \quad D_{t}=\frac{3}{4} \bar{D}, \text { with } \bar{D}=\frac{k_{B} T \ln \{L / D\}}{3 \pi \eta_{0} L} .
$$

Here, $\bar{D}$ is the orientationaly averaged translational diffusion coefficient of a noninteracting, free rod (with $\eta_{0}$ the shear viscosity of the solvent). The orientational dependence of the friction coefficient is described by the tensor $\mathbf{D}$. Here we used that for a very long and thin rod, the friction coefficient for motion parallel to its long axis is twice as small as the friction coefficient for perpendicular motion. The rotational diffusion coefficient $D_{r}$ of a free rod is equal to

$$
D_{r}=\frac{3 k_{B} T \ln \{L / D\}}{\pi \eta_{0} L^{3}} .
$$

Using that,

$$
\begin{aligned}
P(\mathbf{r}, \hat{\mathbf{u}}, t)= & \int d \mathbf{r}_{2} \cdots \int d \mathbf{r}_{N} \oint d \hat{\mathbf{u}}_{2} \cdots \oint d \hat{\mathbf{u}}_{N} \\
& \times P\left(\mathbf{r}, \mathbf{r}_{2}, \ldots, \mathbf{r}_{N}, \hat{\mathbf{u}}, \hat{\mathbf{u}}_{2}, \ldots, \hat{\mathbf{u}}_{N}, t\right),
\end{aligned}
$$

integration of the Smoluchowski equation in principle leads to an equation of motion for the pdf $P(\mathbf{r}, \hat{\mathbf{u}}, t)$ of interest. Assuming pairwise additivity of the total interaction energy of the assembly of $N$ rods, on integration one encounters the two-particle pdf $P_{2}$, which is defined as

$$
\begin{aligned}
P_{2}\left(\mathbf{r}, \mathbf{r}^{\prime}, \hat{\mathbf{u}}, \hat{\mathbf{u}}^{\prime}, t\right)= & \int d \mathbf{r}_{3} \cdots \int d \mathbf{r}_{N} \oint d \hat{\mathbf{u}}_{3} \cdots \oint d \hat{\mathbf{u}}_{N} \\
& \times P\left(\mathbf{r}, \mathbf{r}^{\prime}, \mathbf{r}_{3} \ldots, \mathbf{r}_{N}, \hat{\mathbf{u}}, \hat{\mathbf{u}}^{\prime}, \hat{\mathbf{u}}_{3}, \ldots, \hat{\mathbf{u}}_{N}, t\right),
\end{aligned}
$$

similar to the definition of the single-particle pdf in Eq. (6). This pdf can be expressed in terms of the single-particle pdf of interest as follows. The pair-correlation function $g$ is defined as

$$
P_{2}\left(\mathbf{r}_{1}, \mathbf{r}_{2}, \hat{\mathbf{u}}_{1}, \hat{\mathbf{u}}_{2}, t\right)=P\left(\mathbf{r}_{1}, \hat{\mathbf{u}}_{1}, t\right) P\left(\mathbf{r}_{2}, \hat{\mathbf{u}}_{2}, t\right) g\left(\mathbf{r}_{1}, \mathbf{r}_{2}, \hat{\mathbf{u}}_{1}, \hat{\mathbf{u}}_{2}, t\right) .
$$

For suspensions of very long and thin rods with short-ranged repulsive interactions in equilibrium, the pair-correlation function is simply equal to ( $V$ is the pair-interaction potential between two rods)

$$
g\left(\mathbf{r}-\mathbf{r}^{\prime}, \hat{\mathbf{u}}, \hat{\mathbf{u}}^{\prime}, t\right)=\exp \left\{-\beta V\left(\mathbf{r}-\mathbf{r}^{\prime}, \hat{\mathbf{u}}, \hat{\mathbf{u}}^{\prime}\right)\right\},
$$

up to concentrations where the nematic phase is stable [33-36] For the present case where there is local equilibrium, this form of $g$ will be a good approximation as well. What is neglected in using this form of the correlation function are dynamic correlations $[37,38]$. For the relatively slow dynamics of demixing such correlations are probably not important. Finally noting that for hard-core interactions (with $\boldsymbol{\nabla}$ the gradient operator with respect to $\mathbf{r}$ ),

$$
\begin{aligned}
\exp & \left\{-\beta V\left(\mathbf{r}-\mathbf{r}^{\prime}, \hat{\mathbf{u}}, \hat{\mathbf{u}}^{\prime}\right)\right\} \boldsymbol{\nabla} V\left(\mathbf{r}-\mathbf{r}^{\prime}, \hat{\mathbf{u}}, \hat{\mathbf{u}}^{\prime}\right) \\
& =-\beta^{-1} \boldsymbol{\nabla}\left[\exp \left\{-\beta V\left(\mathbf{r}-\mathbf{r}^{\prime}, \hat{\mathbf{u}}, \hat{\mathbf{u}}^{\prime}\right)\right\}-1\right]
\end{aligned}
$$

and (with $\mathbf{R}=\mathbf{r}_{1}-\mathbf{r}_{2}$ )

$$
\int d \mathbf{R}\left[\exp \left\{-\beta V\left(\mathbf{r}-\mathbf{r}^{\prime}, \hat{\mathbf{u}}, \hat{\mathbf{u}}^{\prime}\right)\right\}-1\right]=-2 D L^{2}\left|\hat{\mathbf{u}} \times \hat{\mathbf{u}}^{\prime}\right|,
$$

it is found by integration of the $N$-particle Smoluchowski equation that 


$$
\begin{aligned}
\frac{\partial}{\partial t} \rho(\mathbf{r}, \hat{\mathbf{u}}, t)= & D_{t} \boldsymbol{\nabla} \cdot \mathbf{D}(\hat{\mathbf{u}}) \cdot\{\boldsymbol{\nabla} \rho(\mathbf{r}, \hat{\mathbf{u}}, t)-\beta \rho(\mathbf{r}, \hat{\mathbf{u}}, t) \overline{\mathbf{F}}(\mathbf{r}, \hat{\mathbf{u}}, t)\} \\
& +D_{r} \hat{\mathcal{R}} \cdot\{\hat{\mathcal{R}} \rho(\mathbf{r}, \hat{\mathbf{u}}, t)-\beta \rho(\mathbf{r}, \hat{\mathbf{u}}, t) \overline{\mathbf{T}}(\mathbf{r}, \hat{\mathbf{u}}, t)\},
\end{aligned}
$$

where the pdf $\rho(\mathbf{r}, \hat{\mathbf{u}}, t)$,

$$
\rho(\mathbf{r}, \hat{\mathbf{u}}, t) \equiv N P(\mathbf{r}, \hat{\mathbf{u}}, t),
$$

is the "number density of rods at $\mathbf{r}$ with orientation $\hat{\mathbf{u}}$," and where the average force $\overline{\mathbf{F}}$ and torque $\overline{\mathbf{T}}$ on a rod with position $\mathbf{r}$ and orientation $\hat{\mathbf{u}}$ due to interactions with other rods are given by

$$
\overline{\mathbf{F}}(\mathbf{r}, \hat{\mathbf{u}}, t)=-\boldsymbol{\nabla} V^{\mathrm{eff}}(\mathbf{r}, \hat{\mathbf{u}}, t) \quad \text { and } \quad \overline{\mathbf{T}}(\mathbf{r}, \hat{\mathbf{u}}, t)=-\hat{\mathcal{R}} V^{\mathrm{eff}}(\mathbf{r}, \hat{\mathbf{u}}, t),
$$

with the effective potential $V^{\text {eff }}$ equal to

$$
\begin{aligned}
V^{\mathrm{eff}}(\mathbf{r}, \hat{\mathbf{u}}, t)= & \frac{1}{2} D L^{2} \beta^{-1} \oint d \hat{\mathbf{u}}^{\prime}\left|\hat{\mathbf{u}} \times \hat{\mathbf{u}}^{\prime}\right| \\
& \times \int_{-1}^{+1} d l \int_{-1}^{+1} d l^{\prime} \rho\left(\mathbf{r}+\frac{1}{2} L l \hat{\mathbf{u}}+\frac{1}{2} L l^{\prime} \hat{\mathbf{u}}^{\prime}, \hat{\mathbf{u}}^{\prime}, t\right) .
\end{aligned}
$$

The equations of motion (11), (13), and (14) have been put forward before in Refs. [29,30]. The present derivation reveals the approximations that are involved in these equations of motion. First of all, the pair-correlation function $g$ is taken equal to the form (8). This form is valid for long and thin rods with short-ranged repulsive interactions in equilibrium [33-36]. Using this form in the present case neglects nonequilibrium effects on $g$ and it neglects dynamic correlations $[37,38]$. Since there is local equilibrium during the initial stage of demixing, nonequilibrium contributions are probably small. As in all previous theoretical treatments, we shall neglect contributions related to dynamic correlations. It is not known yet how to incorporate these correlations. Second, hydrodynamic interactions between rods are neglected. For the long and thin rods under consideration, the volume fraction of interest scales like $D / L$, and is therefore very small. The average distance between "beads" is therefore large, so that hydrodynamic interactions are unimportant for sufficiently long rods.

In order to describe initial decomposition kinetics, the pdf $\rho(\mathbf{r}, \hat{\mathbf{u}}, t)$ is written as

$$
\rho(\mathbf{r}, \hat{\mathbf{u}}, t)=\bar{\rho} P_{0}(\hat{\mathbf{u}}, t)+\delta \rho(\mathbf{r}, \hat{\mathbf{u}}, t),
$$

where $\delta \rho$ is the small deviation with respect to the initial pdf $\bar{\rho} P_{0}(\hat{\mathbf{u}}, t=0)$. Note that $P_{0}$ is generally a function of time, which reflects the temporal evolution of alignment of the otherwise homogeneous system. Substitution of the representation (15) into the equations of motion (11), (13), and (14), linearization with respect to $\delta \rho$ and Fourier transformation leads to two distinct contributions: terms related to the Fourier transform $\delta \rho(\mathbf{k}, \hat{\mathbf{u}}, t)$ of $\delta \rho(\mathbf{r}, \hat{\mathbf{u}}, t)$ and terms proportional to the delta distribution $\delta(\mathbf{k})$ (see Appendix A for details). The latter terms describe the temporal evolution of $P_{0}(\hat{\mathbf{u}}, t)$, that is, the time dependence of the degree of alignment of the otherwise homogeneous system. The former terms lead to

$$
\begin{aligned}
\frac{\partial}{\partial t} \delta \rho(\mathbf{k}, \hat{\mathbf{u}}, t)= & -D_{t} \mathbf{k} \cdot \mathbf{D}(\hat{\mathbf{u}}) \cdot \mathbf{k}\{\delta \rho(\mathbf{k}, \hat{\mathbf{u}}, t) \\
& \left.+4 \pi P_{0}(\hat{\mathbf{u}}, t) \oint d \hat{\mathbf{u}}^{\prime} W\left(\mathbf{k}, \hat{\mathbf{u}}^{\prime}, \hat{\mathbf{u}}^{\prime}\right) \delta \rho\left(\mathbf{k}, \hat{\mathbf{u}}^{\prime}, t\right)\right\} \\
& +D_{r} \hat{\mathcal{R}} \cdot\{\hat{\mathcal{R}} \delta \rho(\mathbf{k}, \hat{\mathbf{u}}, t) \\
& \left.+4 \pi P_{0}(\hat{\mathbf{u}}, t) \hat{\mathcal{R}} \oint d \hat{\mathbf{u}}^{\prime} W\left(\mathbf{k}, \hat{\mathbf{u}}, \hat{\mathbf{u}}^{\prime}\right) \delta \rho\left(\mathbf{k}, \hat{\mathbf{u}}^{\prime}, t\right)\right\} \\
& +\frac{8}{\pi} \frac{L}{D} \varphi D_{r} \hat{\mathcal{R}} \cdot\left\{\delta \rho(\mathbf{k}, \hat{\mathbf{u}}, t) \hat{\mathcal{R}} \oint d \hat{\mathbf{u}}^{\prime}\left|\hat{\mathbf{u}} \times \hat{\mathbf{u}}^{\prime}\right|\right. \\
& \left.\times P_{0}\left(\hat{\mathbf{u}}^{\prime}, t\right)\right\},
\end{aligned}
$$

where the integral kernel $W$ is defined as

$$
W\left(\mathbf{k}, \hat{\mathbf{u}}, \hat{\mathbf{u}}^{\prime}\right)=\frac{2}{\pi^{2}} \frac{L}{D} \varphi\left|\hat{\mathbf{u}} \times \hat{\mathbf{u}}^{\prime}\right| j_{0}\left(\frac{1}{2} L \mathbf{k} \cdot \hat{\mathbf{u}}\right) j_{0}\left(\frac{1}{2} L \mathbf{k} \cdot \hat{\mathbf{u}}^{\prime}\right),
$$

with $j_{0}(x)=\sin \{x\} / x$ and with $\varphi=(\pi / 4) D^{2} L \bar{\rho}$ the volume fraction of rods. Appendix A contains mathematical details on the Fourier transformation leading to these results. Collecting terms $\sim \delta(\mathbf{k})$ leads to the equation of motion for $P_{0}$,

$$
\begin{aligned}
\frac{\partial}{\partial t} P_{0}(\hat{\mathbf{u}}, t)= & D_{r} \hat{\mathcal{R}} \cdot\left\{\hat{\mathcal{R}} P_{0}(\hat{\mathbf{u}}, t)+\frac{8}{\pi} \frac{L}{D} \varphi P_{0}(\hat{\mathbf{u}}, t) \hat{\mathcal{R}} \oint d \hat{\mathbf{u}}^{\prime}\right. \\
& \left.\times\left|\hat{\mathbf{u}} \times \hat{\mathbf{u}}^{\prime}\right| P_{0}\left(\hat{\mathbf{u}}^{\prime}, t\right)\right\} .
\end{aligned}
$$

The isotropic state for which $P_{0} \equiv 1 / 4 \pi$ is a stationary solution of the latter equation of motion for all concentrations. For nonisotropic initial states, the time dependence of $P_{0}(\hat{\mathbf{u}}, t)$ determines demixing kinetics as well, through the appearance of $P_{0}$ in the equation of motion (16) for $\delta \rho(\mathbf{k}, \hat{\mathbf{u}}, t)$. This renders the analysis of demixing kinetics with nonisotropic initial states much more difficult as compared to the isotropic initial state.

In Refs. [25] and [26], the rotational diffusion coefficient $D_{r}$ is interpreted as a concentration dependent diffusion coefficient, which is strongly affected by entanglements. The present derivation shows, however, that the diffusion coefficients $D_{r}$ and $D_{r}$ are those for a free, noninteracting rod. The true, concentration dependent rotational diffusion coefficient must be obtained by solving the above equations of motion explicitly and calculating from that solution the appropriate time-correlation functions. Either one neglects the interaction terms in the above equations of motion in which case $D_{t}$ can be interpreted as a concentration dependent diffusion coefficient, or the interaction terms are taken into account in which case $D_{r}$ is the rotational diffusion coefficient of a noninter- 


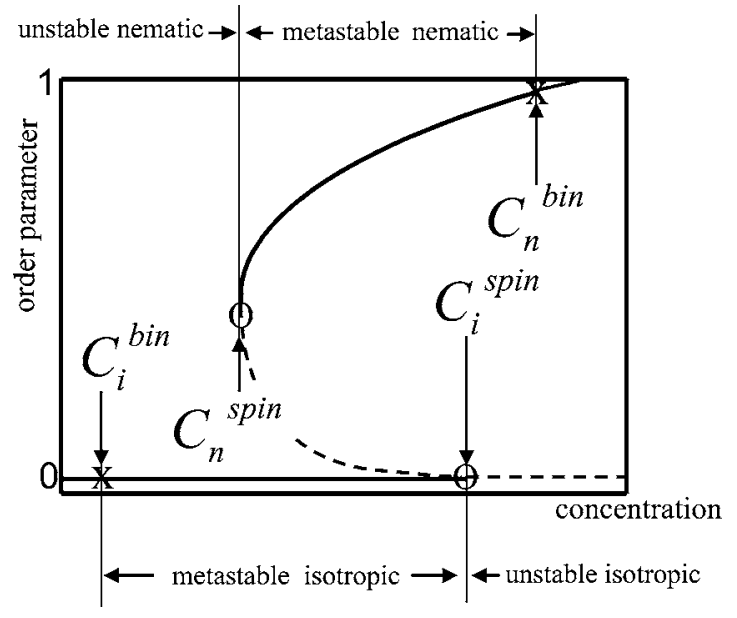

FIG. 1. The bifurcation diagram, where the orientational order parameter $P_{2}$ is plotted against concentration. Indicated are the various regions where different initial states of the homogeneous suspension are unstable. The points marked by $\mathrm{X}$ and $\mathrm{O}$ are spinodal and binodal points, respectively.

acting rod. In analyzing the above equations of motion with the inclusion of the interaction contributions (the terms $\sim \varphi$ ) it is not correct to set $D_{r}$ equal to 0 .

In the following we shall first comment on the instability of initial states and then analyze demixing kinetics with an isotropic initial state. Demixing from a nonisotropic initial state requires the (numerical) solution of Eq. (18), which is beyond the scope of the present paper.

\section{ON THE INSTABILITY OF INITIAL STATES}

A convenient way to analyze the stability of a homogeneous initial state is to derive an equation of motion for the order parameter tensor,

$$
\mathbf{S}_{0}(t)=\oint d \hat{\mathbf{u}} \hat{\mathbf{u}} \hat{\mathbf{u}} P_{0}(\hat{\mathbf{u}}, t),
$$

from Eq. (18). The largest eigenvalue $P_{2}$ of the tensor $\mathbf{Q}_{0}(t)=3 / 2\left[\mathbf{S}_{0}(t)-1 / 3 \hat{\mathbf{I}}\right]$ (where $\hat{\mathbf{I}}$ is the identity) measures the degree of alignment. For the isotropic state $P_{2}=0$, while for a perfectly aligned state $P_{2}=1$. A stability analysis of stationary solutions of this equation of motion is most conveniently made on the basis of a bifurcation diagram [39], where $P_{2}$ for stationary solutions is plotted against the concentration. A schematic bifurcation diagram is given in Fig.1. The two solid lines represent stable stationary solutions of Eq. (18), while the dotted lines represent unstable stationary solutions. The isotropic state ceases to be stable above the concentration indicated as $C_{i}^{\text {spin }}$, while the nematic state becomes unstable at concentrations lower than $C_{n}^{\text {spin }}$. Above $C_{i}^{\text {spin }}$, the isotropic state is still a stationary solution of Eq. (18), but is now unstable. Below $C_{n}^{\text {spin }}$, on the contrary, there is no unstable stationary nematic state. The two spinodal concentrations $C_{i}^{\text {spin }}$ and $C_{n}^{\text {spin }}$ are connected by a separatrix which separates the basins of attraction for the isotropic and nematic state. A homogeneous initial state above this separa- trix develops a higher degree of alignment, while an initial state below the separatrix becomes more isotropic.

Note that the bifurcation diagram relates to homogeneous systems. In an experiment, starting from a homogeneous state, inhomogeneities develop simultaneously with the change of the order parameter of the otherwise homogeneous system. In equilibrium, after completion of phase separation, there is an isotropic phase with concentration $C_{i}^{\text {bin }}$ in coexistence with a nematic phase with concentration $C_{n}^{\text {bin }}$. One can either start from a stationary state, in which case $P_{0}$ in Eq. (16) is independent of time, or from a nonstationary state, like a nematic state with a concentration lower than $C_{n}$, in which case the time dependence of the temporal evolution of alignment of the otherwise homogeneous system couples to the evolution of inhomogeneities through the time dependence of $P_{0}$ in Eq. (16).

One way to prepare an initial state experimentally is to shear a suspension with a shear rate that is large enough to stabilize the system against phase separation (see Refs. [37] and [40] for a discussion of the bifurcation diagram for sheared systems), and then quench to zero shear rate. It is observed in such experiments [41] that spinodal decomposition occurs at lower concentrations, while nucleation and growth is observed at higher concentrations. This observation is in accordance with the above analysis, assuming that the state after shear quenching is still sufficiently aligned at the instant of time that phase separation sets in. For an isotropic initial state this would have been reversed: spinodal decomposition at high concentrations and nucleation and growth at lower concentrations. In case of suspensions of, for example, fd virus, an isotropic initial state can be realized by a pressure quench where water is compressed by about $10 \%$ which increases the fd concentration.

The observed phase separation kinetics thus depends crucially on the preparation of the initial state of the suspension. In the present paper we will analyze phase separation with an isotropic initial state. This is a kind of special state, since it is a stationary solution of the equation of motion (18) for the concentrations above $C_{i}^{\text {spin }}$ of interest. Any other initial state would require the (numerical) solution of Eq. (18) for $P_{0}$, which is beyond the scope of the present paper.

\section{A REPRESENTATION FOR THE PROBABILITY DENSITY FUNCTION (PDF)}

Assuming a moderate degree of orientational order during the initial stage of demixing, $\delta \rho$ will be represented as

$$
\delta \rho(\mathbf{r}, \hat{\mathbf{u}}, t)=A_{0}(\mathbf{r}, t)+\mathbf{A}_{2}(\mathbf{r}, t): \hat{\mathbf{u}} \hat{\mathbf{u}}
$$

where $\mathbf{A}_{2}$ is a symmetric second rank tensor. In order that the form $\mathbf{A}_{2}$ : ûu does not contain scalar terms independent of $\hat{\mathbf{u}}$ that contribute to the first term $A_{0}$, the tensor $\mathbf{A}_{2}$ can be taken traceless. Hence (here and in the following, summation over repeated indices is understood)

$$
A_{2, n n}=0 \text {. }
$$

It follows that the representation (20) can also be written as 


$$
\delta \rho(\mathbf{r}, \hat{\mathbf{u}}, t)=A_{0}(\mathbf{r}, t)+\mathbf{A}_{2}(\mathbf{r}, t): \mathbf{q},
$$

where $\mathbf{q}=\hat{\mathbf{u}} \mathbf{u}-1 / 3 \hat{\mathbf{I}}$. This representation is equivalent to an expansion in spherical harmonics up to second order.

Using that

$$
\begin{gathered}
\oint d \hat{\mathbf{u}} \hat{u}_{i} \hat{u}_{j}=\frac{4 \pi}{3} \delta_{i j}, \\
\oint d \hat{\mathbf{u}} \hat{u}_{i} \hat{u}_{j} \hat{u}_{k} \hat{u}_{l}=\frac{4 \pi}{15}\left[\delta_{i j} \delta_{k l}+\delta_{i k} \delta_{j l}+\delta_{i l} \delta_{j k}\right],
\end{gathered}
$$

it is readily found from Eq. (20) that the spatially varying part of the number density $\delta \rho(\mathbf{r}, t)$ of rods, irrespective of their orientation, is equal to

$$
\delta \rho(\mathbf{r}, t) \equiv \oint d \hat{\mathbf{u}} \delta \rho(\mathbf{r}, \hat{\mathbf{u}}, t)=4 \pi A_{0}(\mathbf{r}, t),
$$

while the orientational order parameter density $\delta \mathbf{s}(\mathbf{r}, t)$ is equal to

$$
\delta \mathbf{s}(\mathbf{r}, t) \equiv \oint d \hat{\mathbf{u}} \hat{\mathbf{u}} \hat{\mathbf{u}} \delta \rho(\mathbf{r}, \hat{\mathbf{u}}, t)=\frac{4 \pi}{3} \hat{\mathbf{I}} A_{0}+\frac{8 \pi}{15} \mathbf{A}_{2} .
$$

The representation (22) can thus alternatively be written as

$$
\delta \rho(\mathbf{r}, \hat{\mathbf{u}}, t)=\frac{7}{8 \pi} \delta \rho(\mathbf{r}, t)+\frac{15}{8 \pi} \delta \mathbf{s}(\mathbf{r}, t): \hat{\mathbf{u}} \hat{\mathbf{u}}
$$

and

$$
\mathbf{A}_{2}=\frac{15}{8 \pi}\left[\delta \mathbf{s}(\mathbf{r}, t)-\frac{1}{3} \hat{\mathbf{I}} \delta \rho(\mathbf{r}, t)\right] .
$$

The contribution $\sim \mathbf{A}_{2}$ to the pdf thus describes the development of orientational order (coupled to the development of inhomogeneities) during demixing. As will turn out, for rod dispersions it is not the number density $A_{0}$ that is unstable, but rather the orientational contribution $\mathbf{A}_{2}$, leading to enslavement of number density by orientational order.

Note that normalization of the pdf in Eq. (20) requires that

$$
\int d \mathbf{r} A_{0}(\mathbf{r}, t)=0
$$

where it it used that $\mathbf{A}_{2}$ is traceless. This relation expresses conservation of the number of rods.

\section{EQUATIONS OF MOTION FOR $A_{0}$ AND $A_{2}$}

The equation of motion (16) and (17) with $P_{0}(\hat{\mathbf{u}}, t)$ $\equiv 1 / 4 \pi$ will be expanded both with respect to gradients in inhomogeneities and with respect to orientational order. The gradient expansion is equivalent to an expansion with respect to the wave vector, while the expansion with respect to orientational order is equivalent to an expansion in terms of orthogonal polyadic products of $\hat{\mathbf{u}}$. We chose to expand with respect to orthogonal polyadic products rather than using spherical harmonics since this leads in a more natural way to explicit expressions for equations of motion for both $A_{0}$ and $\mathbf{A}_{2}$.

The isotropic pdf $P_{0}(\hat{\mathbf{u}}, t) \equiv 1 / 4 \pi$ is the (stable or unstable) stationary solution of Eq. (18) for all concentrations. For an isotropic initial state, Eq. (16) therefore reduces to

$$
\begin{aligned}
\frac{\partial}{\partial t} \delta \rho(\mathbf{k}, \hat{\mathbf{u}}, t)= & \left(-D_{t} \mathbf{k} \cdot \mathbf{D}(\hat{\mathbf{u}}) \cdot \mathbf{k}+D_{r} \hat{\mathcal{R}} \cdot \hat{\mathcal{R}}\right)\{\delta \rho(\mathbf{k}, \hat{\mathbf{u}}, t) \\
& \left.+\oint d \hat{\mathbf{u}}^{\prime} W\left(\mathbf{k}, \hat{\mathbf{u}}, \hat{\mathbf{u}}^{\prime}\right) \delta \rho\left(\mathbf{k}, \hat{\mathbf{u}}^{\prime}, t\right)\right\} .
\end{aligned}
$$

This equation of motion (with $D_{r}$ set equal to 0 ) has been analyzed in Refs. [25,26]. The microscopic derivation in Sec. II reveals the approximations under which this equation of motion is valid [see the discussion just below Eq. (14)]. In addition, it follows from the derivation in Sec. II that this equation of motion can only be used when the initial state is isotropic. Otherwise, the time dependence of $P_{0}(\hat{\mathbf{u}}, t)$ in the more general equation of motion (16) must be kept. In this sense the isotropic state is a kind of special initial state. Other initial states require the (numerical) solution of Eq. (18) as an input to the equation of motion (16) that describes the temporal evolution of inhomogeneities.

The Bessel functions $j_{0}$ in the integral kernel (17) are straightforwardly expanded up to second order in the wave vector k, like in Ref. [25] (the equation of motion with $D_{r}$ $=0$ and using an orientationally pre-averaged translational diffusion coefficient, is solved analytically in Ref. [26] for arbitrary wave vectors). The resulting integral with respect to $\hat{\mathbf{u}}^{\prime}$ in the equation of motion (29) can then be performed explicitly. Expanding in terms of orthogonal polyadic products of $\hat{\mathbf{u}}$ and equation coefficients of the two lowest order polyadic products then leads to the following set of equations for the coefficients in the representation (22) of the density (mathematical details are given in Appendix B):

$$
\begin{aligned}
\frac{\partial}{\partial t} A_{0}= & -\bar{D} k^{2}\left(A_{0}\left\{1+2 \frac{L}{D} \varphi\left[1-\frac{29}{960}(k L)^{2}\right]\right\}\right. \\
& \left.+\frac{1}{10} \mathbf{A}_{2}: \hat{\mathbf{k}} \hat{\mathbf{k}}\left\{1-\frac{1}{4} \frac{L}{D} \varphi\left[1+\frac{29}{84}(k L)^{2}\right]\right\}\right),
\end{aligned}
$$

and

$$
\begin{aligned}
\frac{\partial}{\partial t} \mathbf{A}_{2}= & -6 D_{r}\left(\mathbf{A}_{2}\left\{1-\frac{1}{4} \frac{L}{D} \varphi\left[1-\frac{27}{896}(k L)^{2}\right]\right\}\right. \\
& \left.+\frac{1}{168}(k L)^{2} \frac{L}{D} \varphi\left(\mathbf{A}_{2} \cdot \hat{\mathbf{k}} \hat{\mathbf{k}}+\hat{\mathbf{k}} \hat{\mathbf{k}} \cdot \mathbf{A}_{2}-\frac{2}{3} \hat{\mathbf{I}} \mathbf{A}_{2}: \hat{\mathbf{k}} \hat{\mathbf{k}}\right)\right) \\
& -\bar{D} k^{2}\left(\frac{6}{7} \mathbf{A}_{2}\left\{1-\frac{1}{4} \frac{L}{D} \varphi\left[1-\frac{5}{384}(k L)^{2}\right]\right\}\right. \\
& +\frac{3}{14}\left(\mathbf{A}_{2} \cdot \hat{\mathbf{k}} \hat{\mathbf{k}}+\hat{\mathbf{k}} \hat{\mathbf{k}} \cdot \mathbf{A}_{2}-\frac{2}{3} \hat{\mathbf{I}} \mathbf{A}_{2}: \hat{\mathbf{k}} \hat{\mathbf{k}}\right) \\
& \times\left\{1-\frac{1}{4} \frac{L}{D} \varphi\left[1-\frac{13}{96}(k L)^{2}\right]\right\}+\frac{3}{4} A_{0}\left(\hat{\mathbf{k}} \hat{\mathbf{k}}-\frac{1}{3} \hat{\mathbf{I}}\right) \\
& \times\left\{1+2 \frac{L}{D} \varphi\left[1-\frac{1}{12}(k L)^{2}\right]\right\}-\frac{23}{3476}(k L)^{2} \frac{L}{D} \varphi
\end{aligned}
$$




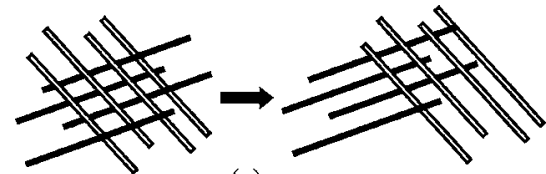

(a)
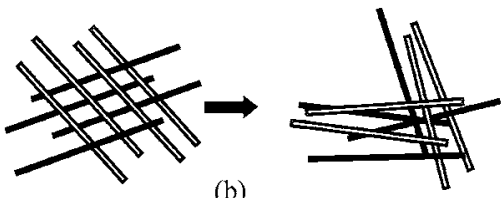

(b)

FIG. 2. Two possible mechanisms for the development of inhomogeneities in orientational order. (a) segregation of rods with different orientational order due to translational diffusion, and (b) pure rotational diffusion.

$$
\left.\times\left(\hat{\mathbf{k}} \hat{\mathbf{k}}-\frac{1}{3} \hat{\mathbf{I}}\right)\left(\mathbf{A}_{2}: \hat{\mathbf{k}} \hat{\mathbf{k}}\right)\right) .
$$

As pointed out in Appendix B, these equations of motion are accurate to within about $5 \%$ for

$$
k L<3 \text {. }
$$

There are two things to be noted about the above equations of motion. First of all, there are two terms in the equation of motion (31) for $\mathbf{A}_{2}$ that render $\mathbf{A}_{2}$ unstable for $(L / D) \varphi>4$, one term $\sim \bar{D} k^{2}$ and one term $\sim 6 D_{r}$. The former term expresses growth of orientational order due to translational diffusion, where rods with different orientation "segregate," while the latter term expresses growth due to re-orientation (see Fig. 2). As will be seen in the section where the relevant contribution to the equations of motion are identified, the rotational contribution leads to much faster demixing as compared to the translational contribution. Demixing is thus dominated by rotational diffusion. Second, the equation of motion for $A_{0}$ is intrinsically stable for all concentrations and wave vectors for which $k L<3$. The density becomes inhomogeneous solely due to its coupling with orientational order as signified by the second term $\sim \mathbf{A}_{2}: \hat{\mathbf{k}} \hat{\mathbf{k}}$ in the equation of motion (30) for $A_{0}$. In other words, the number density of rods is enslaved during demixing by the unstable orientational order parameter. This will be quantified in the section where the equations of motion are solved.

Not all terms in the equations of motion are equally relevant. In the subsequent section, the equations of motion will be simplified by disregarding the irrelevant terms. These simplified equations of motion will subsequently be solved analytically.

\section{SIMPLIFICATION OF THE EQUATIONS OF MOTION}

Not all contributions to Eqs. (30) and (31) are equally important. In the present subsection we shall discuss the relative importance of various contributions. Irrelevant contributions will be neglected in the further analysis of equations of motion. This simplifies the analytical solution considerably. The numerical differences between an exact treatment and the simplified one given below have been checked to be negligible.
First consider Eq. (30) for $A_{0}$. In the first term on the right-hand side $\sim A_{0}$, the contribution $\sim(k L)^{2}$ can be neglected since for $k L \approx 1$ it is much smaller than unity. The similar prefactor in the second term $\sim \mathbf{A}_{2},: \hat{\mathbf{k}} \hat{\mathbf{k}}$ of order $(k L)^{2}$ is not small, and we shall keep this term as it stands.

Second, consider Eq. (31). The importance of the rotational contribution $\sim 6 D_{r}$ as compared to the translational contribution $\sim \bar{D} k^{2}$ to Eq. (31) can be quantified as follows. From the expressions (3) and (4) for $D_{r}$ and $\bar{D}$ it follows that $6 D_{r} / \bar{D} k^{2}=54 /(k L)^{2}$. Since typically $k L \approx 1$, the right-hand side is a large number. Hence the terms $\sim \bar{D} k^{2}$ that are of the same symmetry and of the order of magnitude as compared to similar terms $\sim 6 D_{r}$ can be neglected. All terms $\sim \mathbf{A}_{2}$ and $\sim \bar{D} k^{2}$ can thus be neglected against the corresponding terms multiplied by $6 D_{r}$. The only remaining term $\sim \bar{D} k^{2}$ is therefore the term $\sim A_{0}$. As before, the contribution $\sim(k L)^{2}$ in this term is small compared to unity, and shall be omitted.

These considerations lead to the following set of simplified equations of motion:

$$
\begin{aligned}
\frac{\partial}{\partial t} A_{0}= & -\bar{D} k^{2}\left(A_{0}\left\{1+2 \frac{L}{D} \varphi\right\}+\frac{1}{10} \mathbf{A}_{2}: \hat{\mathbf{k}} \hat{\mathbf{k}}\left\{1-\frac{1}{4} \frac{L}{D} \varphi[1\right.\right. \\
& \left.\left.\left.+\frac{29}{84}(k L)^{2}\right]\right\}\right),
\end{aligned}
$$

and

$$
\begin{aligned}
\frac{\partial}{\partial t} \mathbf{A}_{2}= & -6 D_{r}\left(\mathbf{A}_{2}\left\{1-\frac{1}{4} \frac{L}{D} \varphi\left[1-\frac{27}{896}(k L)^{2}\right]\right\}\right. \\
& \left.+\frac{1}{168}(k L)^{2} \frac{L}{D} \varphi\left(\mathbf{A}_{2} \cdot \hat{\mathbf{k}} \hat{\mathbf{k}}+\hat{\mathbf{k}} \hat{\mathbf{k}} \cdot \mathbf{A}_{2}-\frac{2}{3} \hat{\mathbf{I}} \mathbf{A}_{2}: \hat{\mathbf{k}} \hat{\mathbf{k}}\right)\right) \\
& -\frac{3}{4} \bar{D} k^{2} A_{0}\left(\hat{\mathbf{k}} \hat{\mathbf{k}}-\frac{1}{3} \hat{\mathbf{I}}\right)\left\{1+2 \frac{L}{D} \varphi\right\} .
\end{aligned}
$$

One might consider to neglect the second term $\sim \hat{\mathbf{k}} \hat{\mathbf{k}}: \mathbf{A}_{2}$ in the equation of motion for $A_{0}$ since, as argued before, $(L / D) \varphi$ is close to 4 , and there is a prefactor of $1 / 10$ involved. As will be discussed in the following section where the equations of motion are solved, neglecting this term would predict a homogeneous density $A_{0}$ during demixing, and only orientational order would become inhomogeneous. It is precisely this small contribution that enslaves the density to orientational order, which is the main mechanism to render the number density inhomogeneous during demixing.

Note that when rotational motion is disregarded, that is, when $D_{r}$ is taken equal to zero as in Refs. [25] and [26], the first term in the translational contribution $\sim \bar{D} k^{2}$ in Eq. (31) renders the system unstable. As shown above, this contribution is relatively unimportant as compared to the corresponding term resulting from rotational diffusion.

The equations of motion (33) and (34) will be analyzed in the following section in order to predict the time- and wavevector dependence of the scattered intensity, which is the most relevant experimental quantity for demixing suspensions. 


\section{Solution of the equations of motion}

As will be shown in the next section, the scattered intensity is related to $A_{0}$ and $\hat{\mathbf{k}} \hat{\mathbf{k}}: \mathbf{A}_{2}$. We shall therefore derive here explicit equations for these two quantities from the equations of motion (33) and (34). A double contraction of both sides of Eq. (34) with $\hat{\mathbf{k}} \hat{\mathbf{k}}$ leads straightforwardly to the relevant coupled equations of motion for $A_{0}$ and $\hat{\mathbf{k}} \hat{\mathbf{k}}: \mathbf{A}_{2}$. For brevity let us define the vector,

$$
\mathbf{V}(\mathbf{k}, t) \equiv\left(\begin{array}{c}
A_{0}(\mathbf{k}, t) \\
\hat{\mathbf{k}} \hat{\mathbf{k}}: \mathbf{A}_{2}(\mathbf{k}, t)
\end{array}\right) .
$$

The equation of motion for this vector is written as

$$
\frac{\partial}{\partial t} \mathbf{V}=-\left(\begin{array}{cc}
D_{11} k^{2} & D_{12} k^{2} \\
D_{21} k^{2} & D_{22}
\end{array}\right) \cdot \mathbf{V},
$$

where the various diffusion coefficients are equal to

$$
\begin{gathered}
D_{11}=\bar{D}\left\{1+2 \frac{L}{D} \varphi\right\}, \\
D_{12}=\frac{1}{10} \bar{D}\left\{1-\frac{1}{4} \frac{L}{D} \varphi\left[1+\frac{29}{84}(k L)^{2}\right]\right\}, \\
D_{21}=\frac{1}{2} \bar{D}\left\{1+2 \frac{L}{D} \varphi\right\}=\frac{1}{2} D_{11}, \\
D_{22}=6 D_{r}\left\{1-\frac{1}{4} \frac{L}{D} \varphi\left[1-\frac{499}{8064}(k L)^{2}\right]\right\} .
\end{gathered}
$$

As pointed out in the section where the equations of motion (30) and (31) are simplified, the magnitude of $D_{12} k^{2}$ is small in comparison to all other matrix elements in Eq. (36) for three reasons: it is $\sim \bar{D} k^{2}$ and $\sim[1-1 / 4 L / D \varphi(\cdots)]$, and is in addition multiplied by a small prefactor $1 / 10$. If one would neglect the contribution arising from $D_{12}$, that is if $D_{12}$ would be set equal to 0 , then the two eigenvalues of the tensor in Eq. (36) are simply $D_{11}$ and $D_{22}$. This leads to the conclusion that the density $A_{0}$ is always homogeneous since $D_{11}>0$ for all concentrations and wave vectors, and only the orientational contribution $\mathbf{A}_{2}$ to the pdf develops inhomogeneities. The small diffusion coefficient $D_{12}$ thus reflects enslavement of density to orientational order: the number density becomes inhomogeneous because it is "dragged along" by the unstable orientational order. In the analysis of the equation of motion (36) we shall therefore expand eigenvalues with respect to $D_{12} k^{2}$. Using that $D_{21}=D_{11} / 2$ the following expressions for the eigenvalues $\lambda^{( \pm)}$of the diffusion tensor in Eq. (36) are found to leading order in $D_{12} k^{2}$ :

$$
\begin{gathered}
\lambda^{(-)}=D_{22}-\frac{1}{2} \frac{D_{11} D_{12}}{D_{11} k^{2}-D_{22}} k^{4}, \\
\lambda^{(+)}=D_{11} k^{2}+\frac{1}{2} \frac{D_{11} D_{12}}{D_{11} k^{2}-D_{22}} k^{4} .
\end{gathered}
$$

Note that for the concentrations and wave vectors of interest $\left(D_{11} k^{2}-D_{22}\right)>0$. Expanding each of the entries of the corre-
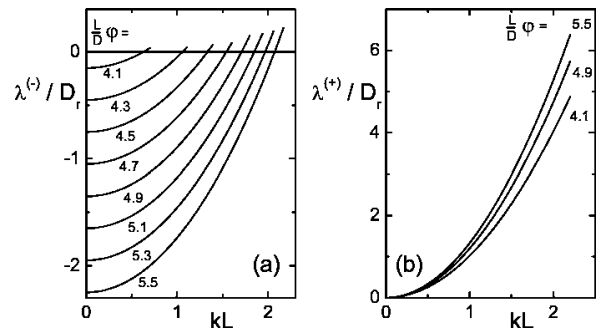

FIG. 3. The eigenvalues $\lambda^{(-)}$(a) and $\lambda^{(+)}$(b) in units of $D_{r}$ as functions of the wave vector for various concentrations as indicated in the figure.

sponding (normalized) eigenvectors $\hat{\mathbf{e}}^{ \pm}$to leading order in $D_{12} k^{2}$, gives

$$
\begin{gathered}
\hat{\mathbf{e}}^{(-)}=\frac{1}{D_{11} k^{2}-D_{22}}\left(\begin{array}{c}
-D_{12} k^{2} \\
D_{11} k^{2}-D_{22}
\end{array}\right), \\
\hat{\mathbf{e}}^{(+)}=\frac{1}{\sqrt{\left(D_{11} k^{2}-D_{22}\right)^{2}+\frac{1}{4} D_{11}^{2} k^{4}}}\left(\begin{array}{c}
D_{11} k^{2}-D_{22} \\
\frac{1}{2} D_{11} k^{2}
\end{array}\right) .
\end{gathered}
$$

The solution to the equation of motion (36) is given by

$$
\mathbf{V}(\mathbf{k}, t)=a^{(-)} \hat{\mathbf{e}}^{(-)} \exp \left\{-\lambda^{(-)} t\right\}+a^{(+)} \hat{\mathbf{e}}^{(+)} \exp \left\{-\lambda^{(+)} t\right\},
$$

where the coefficients $a^{( \pm)}$are to be chosen according to a specified initial value for $\mathbf{V}$. The eigenvalues in Eq. (38) are plotted as functions of the wave vector for several concentrations within the unstable region in Fig. 3 [here it is used that $\bar{D} k^{2} / D_{r}=(k L)^{2} / 9$, which follows from Eqs. (3) and (4)]. Note that the eigenvalue $\lambda^{(-)}$of the unstable mode $\hat{\mathbf{e}}^{(-)}$does not exhibit a minimum at finite wave vectors, as in case of gas-liquid demixing of spherical colloids. This is due to the fact that the nonconserved orientational order is the most prominent unstable quantity, so that an overall prefactor $k^{2}$ to the eigenvalue related to the unstable mode is missing here. As will be seen later, the scattered intensity nevertheless exhibits a maximum at finite wave vectors, which is due to the wave-vector dependence of the prefactors of the time exponentials in Eq. (40).

Without any of the simplifications discussed in the previous section, the above analytical treatment is feasible as well, except that the expressions (37) for the diffusion coefficients become more involved. These expressions are given in Appendix $\mathrm{C}$. The corresponding eigenvalues are essentially undistinguishable from those plotted in Fig. 3. This validates our simplification of the equations of motion.

The only mode of interest for demixing is the unstable mode $\hat{\mathbf{e}}^{(-)}$. Disregarding the contribution $\sim \hat{\mathbf{e}}^{(+)}$in Eq. (40) thus leads to the following solution of the equations of motion:

$$
A_{0}(\mathbf{k}, t)=-\frac{a^{(-)} D_{12} k^{2}}{D_{11} k^{2}-D_{22}} \exp \left\{-\lambda^{(-)} t\right\}
$$




$$
\hat{\mathbf{k}} \hat{\mathbf{k}}: \mathbf{A}_{2}(\mathbf{k}, t)=a^{(-)} \exp \left\{-\lambda^{(-)} t\right\} .
$$

Note that the density $A_{0}$ is proportional to $D_{12} k^{2}$. As stated before, if the small contribution $\sim D_{12} k^{2}$ to the equation of motion for $A_{0}$ that couples $A_{0}$ to $\mathbf{A}_{2}$ would have been set equal to 0 , the density $A_{0}$ would be simply equal to 0 , that is, the number density of rods would be homogeneous during demixing. The small but finite prefactor in the expression (41) for $A_{0}$ thus expresses enslaving of the density to the orientational contribution $\mathbf{A}_{2}$ to the probability density function.
The demixing times of interest are of the order $\tau$ $\approx 1 /\left|D_{22}(k=0)\right|$. As shown in Fig. 4 , the contributions $\sim D_{12}$ to the eigenvalues, multiplied by $1 /\left|D_{22}(k=0)\right|$, are very small as compared to unity. The contributions $\sim D_{12}$ in the expression for the eigenvalues in the time exponents can therefore be neglected. The only importance of the finite value of $D_{12}$ is that it properly describes the enslaving of $A_{0}$ to the orientational contribution of the probability density function by the finite prefactor in Eq. (41) for $A_{0}$. We thus finally arrive at the following solutions of the equations of motion:

$$
\begin{aligned}
& A_{0}(\mathbf{k}, t)=-\frac{\frac{1}{10} \bar{D} k^{2}\left\{1-\frac{1 L}{4 D} \varphi\left[1+\frac{29}{84}(k L)^{2}\right]\right\}}{\bar{D} k^{2}\left\{1+2 \frac{L}{D} \varphi\right\}-6 D_{r}\left\{1-\frac{1 L}{4} \varphi\left[1-\frac{499}{8064}(k L)^{2}\right]\right\}} \hat{\mathbf{k}} \hat{\mathbf{k}}: \mathbf{A}_{2}(\mathbf{k}, t), \\
& \hat{\mathbf{k}} \hat{\mathbf{k}}: \mathbf{A}_{2}(\mathbf{k}, t)=\hat{\mathbf{k}} \hat{\mathbf{k}}: \mathbf{A}_{2}(\mathbf{k}, t=0) \exp \left(-6 D_{r}\left\{1-\frac{1}{4} \frac{L}{D} \varphi\left[1-\frac{499}{8064}(k L)^{2}\right]\right\} t\right) .
\end{aligned}
$$

These expressions will be used in the following section where light scattering during demixing is considered on the basis of Eq. (46) for the scattered intensity.

Note that the critical wave vector $k_{c}$ above which the system becomes stable, that is, where $D_{22}(k)$ becomes positive, is equal to

$$
k_{c} L=2 \sqrt{\frac{8064}{499}} \sqrt{\frac{1}{4}-\frac{1}{\frac{L}{D} \varphi}} \approx 8 \sqrt{\frac{1}{4}-\frac{1}{\frac{L}{D} \varphi}} .
$$

For shallow quenches, that is, for concentrations where the $(L / D) \varphi$ is close to 4 , the critical wave vector is thus relatively small, as can also be seen from Fig. 3. That is, shallow quenches result in relatively large scale inhomogeneities while deeper quenches give rise to relatively small scale inhomogeneities. Since the wave-vector range where the present theory is valid is limited to $k L<3$, it follows that the concentration is limited to

$$
\frac{L}{D} \varphi<9,
$$

which is well within the nematic phase. For even higher concentrations, one should account for the full wave-vector dependence of the integral in the equation of motion (8), which is possible by the same method described here, where, however, wave-vector dependent functions should be determined by numerical integration.

\section{SCATTERED INTENSITY DURING DEMIXING}

The quantity that is of experimental interest is the time resolved scattered intensity at small scattering angles. For the small angles of interest, the scattered intensity is shown in Appendix D to be given by

$$
I \sim\left[\oint d \hat{\mathbf{u}} \rho(\mathbf{k}, \hat{\mathbf{u}}, t) j_{0}\left(\frac{1}{2} L \mathbf{k} \cdot \hat{\mathbf{u}}\right)\right]^{2} .
$$

Here, it is assumed that rods are identical (all rods have the same length and thickness) and that the refractive index difference between the rod and solvent is large as compared to

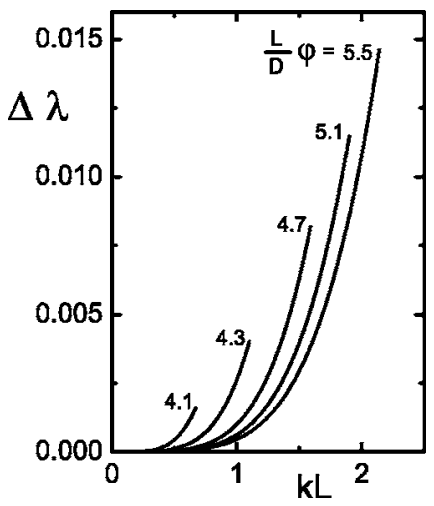

FIG. 4. The contribution $\Delta \lambda \equiv 1 / 2 D_{11} D_{12} k^{4} /\left(D_{11} k^{2}-D_{22}\right) t$ to $\lambda^{(-)} t$ with $t=1 / D_{22}(k=0)$ for various concentrations, as indicated in the figure, as a function of the wave vector for the relevant wavevector range where $\lambda^{(-)}<0$ (see Fig. 3). 

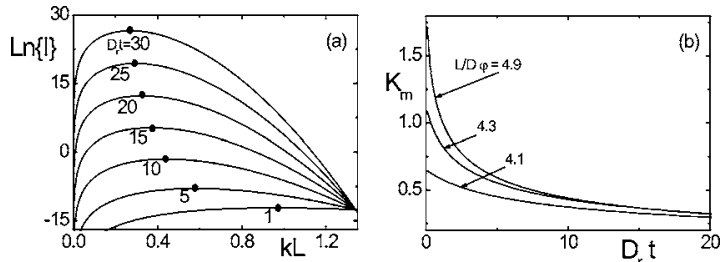

FIG. 5. (a) The (log of the) scattered intensity, apart from the prefactor $\left[\hat{\mathbf{k}} \hat{\mathbf{k}}: \mathbf{A}_{2}(\mathbf{k}, t=0)\right]^{2}$, as a function of the scattering wave vector for $(L / D) \varphi=4.5$ for various dimensionless times $D_{r} t$, as indicated in the figure. The dots indicate the maximum in the scattered intensity. (b) The wave vector $k_{m}$ where the maximum in the scattered occurs as a function of time for three different concentrations, as indicated in the figure.

the difference in refractive index along and perpendicular to the long axis of the rod. Thus depolarized contributions to the scattered intensity are assumed to be negligibly small.

Substitution of the representation (22) into Eq. (45) readily leads to [apart from a factor $(4 \pi)^{2}$ ],

$$
\begin{aligned}
I \sim & \left\{A_{0}(\mathbf{k}, t)\left[1-\frac{1}{72}(k L)^{2}\right]-\frac{1}{180}(k L)^{2} \hat{\mathbf{k}} \hat{\mathbf{k}}: \mathbf{A}_{2}(\mathbf{k}, t)\right. \\
& \left.\times\left[1-\frac{3}{560}(k L)^{2}\right]+\mathcal{O}\left[(k L)^{6}\right]\right\}^{2},
\end{aligned}
$$

where the identities (B6) and (B7) have been used to perform the integrations with respect to $\hat{\mathbf{u}}$. The relevant quantities to describe light scattering experiments are thus $A_{0}$ and $\hat{\mathbf{k}} \hat{\mathbf{k}}: \mathbf{A}_{2}$, which can be obtained from the equations of motion (33) and (34).

Like for gas-liquid spinodal demixing suspensions of spheres, the scattered intensity during isotropic-nematic demixing suspensions of rods is a ringlike pattern, where the intensity-growth rate exhibits a maximum at a particular finite wave vector, as can be seen from Fig. 5(a). This figure is obtained from Eqs. (42) and (46). The occurrence of a maximum in the scattered intensity at finite wave vectors during demixing has a fundamentally different origin for demixing of rods as compared to spheres. For gas-liquid demixing of suspensions of spheres, the eigenvalue (here referred to as $\left.\lambda^{(-)}\right)$itself exhibits an extremum at a finite wave vector. For gas-liquid demixing, $\lambda^{(-)}$is of the form $D k^{2}\left[1-\alpha k^{2}\right]$, where $D$ and $\alpha$ are wave-vector-independent, positive coefficients. The prefactor $k^{2}$ signifies the fact that diffusion of spheres over long distances takes longer times, while $\alpha k^{2}$ signifies the stabilization of large concentrations gradients, which would increase the free energy considerably. It is easily verified that an eigenvalue of this form exhibits an extremum at a finite wave vector. For gas-liquid demixing of suspensions of spheres this maximum in the growth rate results in the maximum in the scattering pattern. As has been discussed in the previous section, for the isotropic-nematic demixing of suspensions of rods, the eigenvalue is of the form $D[1$ $\left.-\alpha k^{2}\right]$, that is, the prefactor $k^{2}$ as compared to spheres is missing here. This results in a wave-vector dependence as given in Fig. 3(a), where there is no extremum of the eigenvalue. This difference in wave-vector dependence of the ei- genvalue $\lambda^{(-)}$for gas-liquid and isotropic-nematic demixing is due to the fact that gas-liquid demixing is governed by translational diffusion while isotropic-nematic demixing is (predominantly) governed by rotational diffusion. The maximum in the scattering pattern is now due to the combination of the wave-vector dependence of the eigenvalue and the wave-vector dependent prefactors to the time exponent. Note that according to Eq. (46), the prefactor of the time exponent is indeed $\sim k^{2}$, rendering the scattered intensity equal to zero at zero wave vector, which expresses conservation of the number of rods.

In Ref. [42], spinodal decomposition kinetics of polymerrod mixtures is described on the basis of time-dependent Landau-Ginzburg equations. Here, rotational and translational diffusion dominated demixing can occur, depending on the quench parameters.

The way to test experimentally whether isotropic-nematic demixing is indeed governed by orientational diffusion is as follows. According to Eqs. (42) and (46), the scattered intensity is $\sim \exp \left\{\lambda^{(-)} t\right\}$. Hence

$$
\frac{\partial}{\partial t} \ln \{I(k, t)\}=2 \lambda^{(-)}=12 D_{r}\left\{1-\frac{1}{4} \frac{L}{D} \varphi\left[1-\frac{499}{8064}(k L)^{2}\right]\right\} .
$$

The slope of a plot of $\ln \{I(k, t)\}$ as a function of $t$ for a given wave vector is thus equal to $\lambda^{(-)}$for that particular wave vector. Repeating this for various wave vectors allows us to construct the wave-vector dependence of $\lambda^{(-)}$. When isotropic-nematic demixing is governed by rotational diffusion, the thus obtained experimental values for $\lambda^{(-)}$should remain finite at zero wave vectors, as in Fig. 3(a).

The time dependence of $k_{m}(t)$ is plotted in Fig. 5(b) for various concentrations. As discussed above, contrary to isotropic-nematic demixing, $k_{m}$ is time independent in the initial stage of gas-liquid demixing.

\section{SUMMARY AND CONCLUSIONS}

An alternative derivation of a well known equation of motion for the probability density function (pdf) of the position and orientation of a rod is presented on the basis of the $N$-particle Smoluchowski equation. This microscopic starting point reveals the approximations under which this equation of motion is valid. The rods should be very long and thin with short-ranged repulsive interactions. Furthermore, hydrodynamic interactions between rods as well as nonequilibrium contributions to the pair-correlation function and dynamic correlations are assumed to negligible. The equation of motion for the pdf of the position and orientation of a rod is expanded with respect to gradients in inhomogeneities of number density and orientational order and is expanded to leading order in orientational order. A number of terms in the resulting equation of motion are shown to be irrelevant. It is shown that the number density of rods is enslaved to orientational order and that demixing is predominantly driven by rotational diffusion. Translational diffusion, where inhomogeneities in orientational order develop due to segregation of rods with different orientation, is shown to be much slower 
than the development of these inhomogeneities due to rotational diffusion. As a result, the eigenvalue of the unstable mode (the "effective rotational diffusion coefficient") remains finite at zero wave vector, contrary to what is known for gas-liquid demixing. The temporal evolution and wavevector dependence of the scattered intensity from a demixing suspension of rods is calculated analytically. Despite the fact that rotational diffusion drives demixing, the scattered intensity exhibits a ringlike scattering pattern. The maximum at finite wave vectors is now not due to a minimum of the wave-vector dependent "effective diffusion coefficient" as for gas liquid demixing, but is due to wave-vector dependent prefactors to the time dependent exponent. This results in a marked time dependence of the location of the wave vector $k_{m}$ where the intensity exhibits its maximum, contrary to gas-liquid demixing where $k_{m}$ is independent of time during the initial stage of demixing.

\section{APPENDIX A: DERIVATION OF EQS. (16) and (17)}

One of the terms encountered on linearization of $\beta \rho(\mathbf{r}, \hat{\mathbf{u}}, t) \overline{\mathbf{T}}(\mathbf{r}, \hat{\mathbf{u}}, t)$ reads

$$
\begin{aligned}
I(\mathbf{r}) \equiv & \frac{1}{2} D L^{2} \bar{\rho} P_{0}(\hat{\mathbf{u}}, t) \hat{\mathcal{R}} \oint d \hat{\mathbf{u}}^{\prime}\left|\hat{\mathbf{u}} \times \hat{\mathbf{u}}^{\prime}\right| \int_{-1}^{+1} d l \int_{-1}^{+1} d l^{\prime} \\
& \times \delta \rho\left(\mathbf{r}+\frac{1}{2} L l \hat{\mathbf{u}}+\frac{1}{2} L l^{\prime} \hat{\mathbf{u}}^{\prime}, \hat{\mathbf{u}}^{\prime}, t\right) .
\end{aligned}
$$

The Fourier transform of the density in the integral can be written as

$$
\begin{gathered}
\int d \mathbf{r} \delta \rho\left(\mathbf{r}+\frac{1}{2} L l \hat{\mathbf{u}}+\frac{1}{2} L l^{\prime} \hat{\mathbf{u}}^{\prime}, \hat{\mathbf{u}}^{\prime}, t\right) \exp \{-i \mathbf{k} \cdot \mathbf{r}\} \\
=\delta \rho\left(\mathbf{k}, \hat{\mathbf{u}}^{\prime}, t\right) \exp \left[i \mathbf{k} \cdot\left(\frac{1}{2} L l \hat{\mathbf{u}}+\frac{1}{2} L l^{\prime} \hat{\mathbf{u}}^{\prime}\right)\right],
\end{gathered}
$$

where the Fourier transform $f(\mathbf{k})$ of a function $f(\mathbf{r})$ is defined as

$$
f(\mathbf{k}) \equiv \int d \mathbf{r} f(\mathbf{r}) \exp \{-i \mathbf{k} \cdot \mathbf{r}\}
$$

Here, the argument of the function indicates whether the function itself or its Fourier transform is meant. Using that

$$
\int_{-1}^{+1} d l \exp \left\{i \frac{1}{2} L l \mathbf{k} \cdot \hat{\mathbf{u}}\right\}=2 \frac{\sin \left\{\frac{1}{2} L \mathbf{k} \cdot \hat{\mathbf{u}}\right\}}{\frac{1}{2} L \mathbf{k} \cdot \hat{\mathbf{u}}} \equiv 2 j_{0}\left(\frac{1}{2} L \mathbf{k} \cdot \hat{\mathbf{u}}\right)
$$

and similarly for the $l^{\prime}$ integration, immediately leads to the result in Eqs. (16) and (17).

\section{APPENDIX B: DERIVATION OF THE SET OF EQS. (30) and (31)}

For the long wavelength spatial variations in concentration and orientational order parameter, it suffices to expand the kernel up to order $(k L)^{2}$. Using that $j_{0}(x)=1-1 / 6 x^{2}$ $+\cdots$ for small $x$ gives

$$
j_{0}\left(\frac{1}{2} L \mathbf{k} \cdot \hat{\mathbf{u}}\right)=F(k L)+G(k L) \hat{\mathbf{k}} \hat{\mathbf{k}}: \mathbf{q}+\mathcal{O}\left[(k L)^{4}\right],
$$

and similarly for $j_{0}\left(1 / 2 L \mathbf{k} \cdot \hat{\mathbf{u}}^{\prime}\right)$. Here $\hat{\mathbf{k}}=\mathbf{k} / k$ is the unit vector along $\mathbf{k}$ and

$$
\begin{aligned}
& F(x)=1-\frac{x^{2}}{72}, \\
& G(x)=-\frac{x^{2}}{24} .
\end{aligned}
$$

This expansion is accurate up to about $5 \%$ for $k L<3$. As will be seen later, this is well within the wave-vector range of interest. The integral kernel is thus written as (the $k L$ dependence of $F$ and $G$ are not denoted here for convenience)

$W\left(\mathbf{k}, \hat{\mathbf{u}}, \hat{\mathbf{u}}^{\prime}\right)=\frac{2}{\pi^{2}} \frac{L}{D} \varphi\left\{F^{2}+G \hat{\mathbf{k}} \hat{\mathbf{k}}:\left(\mathbf{q}+\mathbf{q}^{\prime}\right)+\mathcal{O}\left[(k L)^{4}\right]\right\}\left|\hat{\mathbf{u}} \times \hat{\mathbf{u}}^{\prime}\right|$

The integral in Eq.(16) can be obtained from the relations

$$
\begin{gathered}
\oint d \hat{\mathbf{u}}^{\prime}\left|\hat{\mathbf{u}} \times \hat{\mathbf{u}}^{\prime}\right|=\pi^{2}, \\
\oint d \hat{\mathbf{u}}^{\prime}\left|\hat{\mathbf{u}} \times \hat{\mathbf{u}}^{\prime}\right| \hat{u}_{i}^{\prime} \hat{u}_{j}^{\prime}=\frac{3 \pi^{2}}{8}\left[\delta_{i j}-\frac{1}{3} \hat{u}_{i} \hat{u}_{j}\right], \\
\oint d \hat{\mathbf{u}}^{\prime}\left|\hat{\mathbf{u}} \times \hat{\mathbf{u}}^{\prime}\right| \hat{u}_{i}^{\prime} \hat{u}_{j}^{\prime} \hat{u}_{m}^{\prime} \hat{u}_{n}^{\prime} \\
=-\frac{\pi^{2}}{64} \hat{u}_{i} \hat{u}_{j} \hat{u}_{m} \hat{u}_{n}-\frac{\pi^{2}}{64}\left[\delta_{i j} \hat{u}_{m} \hat{u}_{n}+\delta_{i n} \hat{u}_{j} \hat{u}_{m}\right. \\
\left.+\delta_{i m} \hat{u}_{j} \hat{u}_{n}+\delta_{j n} \hat{u}_{i} \hat{u}_{m}+\delta_{j m} \hat{u}_{i} \hat{u}_{n}+\delta_{m n} \hat{u}_{i} \hat{u}_{j}\right] \\
+\frac{5 \pi^{2}}{64}\left[\delta_{i j} \delta_{m n}+\delta_{i n} \delta_{j m}+\delta_{i m} \delta_{j n}\right] .
\end{gathered}
$$

Substitution of the representation (22) for the pdf and integration thus gives

$$
\begin{aligned}
& \oint d \hat{\mathbf{u}}^{\prime} W\left(\mathbf{k}, \hat{\mathbf{u}}, \hat{\mathbf{u}}^{\prime}\right) \delta \rho\left(\mathbf{k}, \hat{\mathbf{u}}^{\prime}, t\right) \\
&=2 \frac{L}{D} \varphi\left(F^{2} A_{0}+\frac{13}{96} G \mathbf{A}_{2}: \hat{\mathbf{k}} \hat{\mathbf{k}}+\mathbf{q}:\left[\left(-\frac{1}{8} F^{2}+\frac{1}{48} G\right) \mathbf{A}_{2}\right.\right. \\
&\left.+\frac{7}{8} G A_{0} \hat{\mathbf{k}} \hat{\mathbf{k}}-\frac{1}{16} G\left(\mathbf{A}_{2} \cdot \hat{\mathbf{k}} \hat{\mathbf{k}}\right)\right]-\frac{9}{64} G\left(\hat{\mathbf{k}} \hat{\mathbf{k}}: \mathbf{q q}: \mathbf{A}_{2}\right) \\
&\left.+\mathcal{O}\left[(k L)^{4}\right]\right) .
\end{aligned}
$$

In order to be able to equate coefficients in an expansion with respect to the order parameter, we have to construct an orthogonal set of polyadic products of $\hat{\mathbf{u}}$ 's, up to the fourth 
order products that appear in Eq. (16). Integrals to be used to construct such an orthogonal set are

$$
\begin{gathered}
\oint d \hat{\mathbf{u}} \hat{u}_{i} \hat{u}_{j}=\frac{4 \pi}{3} C_{i j}^{(2)}, \\
\oint d \hat{\mathbf{u}} \hat{u}_{i} \hat{u}_{j} \hat{u}_{k} \hat{u}_{l}=\frac{4 \pi}{15} C_{i j k l}^{(4)}, \\
\oint d \hat{\mathbf{u}} \hat{u}_{i} \hat{u}_{j} \hat{u}_{k} \hat{u}_{l} \hat{u}_{m} \hat{u}_{n}=\frac{4 \pi}{105} C_{i j k l m n}^{(6)} .
\end{gathered}
$$

where $C_{n_{1}, \ldots, n_{2 M}}^{(2 M)}$ is the sum of products of $M$ Kronecker deltas with all possible independent combinations of indices. The first few of these coefficients are equal to

$$
\begin{gathered}
C_{i j}^{(2)}=\delta_{i j}, \\
C_{i j k l}^{(4)}=\left[\delta_{i j} \delta_{k l}+\delta_{i k} \delta_{j l}+\delta_{i l} \delta_{j k}\right], \\
C_{i j k l m n}^{(6)}=\left[\delta_{i j}\left\{\delta_{k l} \delta_{m n}+\delta_{k m} \delta_{l n}+\delta_{k n} \delta_{l m}\right\}+\delta_{i k}\left\{\delta_{j l} \delta_{m n}+\delta_{j m} \delta_{l n}\right.\right. \\
\left.+\delta_{j n} \delta_{l m}\right\}+\delta_{i l}\left\{\delta_{j k} \delta_{m n}+\delta_{j m} \delta_{k n}+\delta_{j n} \delta_{k m}\right\}+\delta_{i m}\left\{\delta_{j k} \delta_{l n}\right. \\
\left.\left.+\delta_{j l} \delta_{k n}+\delta_{j n} \delta_{k l}\right\}+\delta_{i n}\left\{\delta_{j k} \delta_{l m}+\delta_{j l} \delta_{k m}+\delta_{j m} \delta_{k l}\right\}\right] .
\end{gathered}
$$

Higher order coefficients can be found from the recursion relation

$$
C_{n_{1}, \ldots, n_{2 M+2}}^{(2 M+2)}=\sum_{j=2}^{2 M+2} \delta_{n_{1} n_{j}} C_{n_{1}, \ldots, n_{j-1}, n_{j+1}, \ldots n_{2 M}}^{(2 M)} .
$$

The expression for $C^{(6)}$ in Eq. (B7) is explicitly written in this form. The prefactor of the coefficient $C^{(2 M)}$ in Eq. (B6) is simply equal to $\oint d \hat{\mathbf{u}} u_{z}^{2 M}=2 \pi \int_{-1}^{+1} d z z^{2 M}=4 \pi /(2 M+1)$, divided by the number of terms in the expression for $C^{(2 M)}$. This number of terms follows from the recursion relation (B8) being equal to $(2 M-1)$ !!. The prefactor of the coefficients is thus equal to $4 \pi /(2 M+1)$ !!. With the above identities, the first three orthogonal polyadic products of $\hat{\mathbf{u}}$ 's are found to be given by

$$
\begin{gathered}
q_{0}=1, \\
q_{2, i j} \equiv q_{i j}=\hat{u}_{i} \hat{u}_{j}-\frac{1}{3} \delta_{i j}, \\
q_{4, i j m n}=\hat{u}_{i} \hat{u}_{j} \hat{u}_{m} \hat{u}_{m}-\frac{1}{7}\left\{\hat{u}_{i} \hat{u}_{j} \delta_{m n}+\hat{u}_{i} \hat{u}_{m} \delta_{j n}+\hat{u}_{i} \hat{u}_{n} \delta_{j m}+\hat{u}_{j} \hat{u}_{m} \delta_{i n}\right. \\
\left.+\hat{u}_{m} \hat{u}_{n} \delta_{i j}+\hat{u}_{j} \hat{u}_{n} \delta_{i m}\right\}+\frac{1}{35}\left\{\delta_{i j} \delta_{m n}+\delta_{i m} \delta_{j n}+\delta_{i n} \delta_{j m}\right\} .
\end{gathered}
$$

These expressions are orthogonal in the sense that

$$
\oint d \hat{\mathbf{u}} \mathbf{q}_{k} \mathbf{q}_{l}=\mathbf{0}, \quad \text { when } k \neq l .
$$

The tensors $q_{0}, \mathbf{q}_{2}$, and $\mathbf{q}_{4}$ are the polyadic equivalent of zeroth, second, and fourth order spherical harmonics, respec- tively. Once an expression of the form (where $\odot$ stands for fourfold contraction)

$$
0=q_{0} R+\mathbf{q}_{2}: \mathbf{S}+\mathbf{q}_{4} \odot \mathbf{T}+\cdots=R+\mathbf{q}: \mathbf{S}+\mathbf{q}_{4} \odot \mathbf{T}+\cdots
$$

is found, it follows by integration and the orthogonality relations (B10) that $R=0$. Furthermore, multiplying both sides of Eq. (B11) with $\mathbf{q}$ and integrating gives

$$
\oint d \hat{\mathbf{u} q q}: \mathbf{S}=\mathbf{0} .
$$

Since $\mathbf{S}$ can be taken traceless and symmetric, it follows from Eq. (B6) that

$$
\mathbf{S}=\mathbf{0} .
$$

This allows us to obtain the equation of motion for $\mathbf{A}_{2}$, where $\mathbf{S}$ is the sum of all terms $\sim \mathbf{q}$ in an expansion with respect to

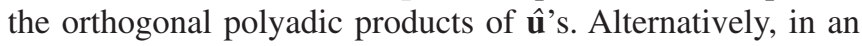
expansion of an arbitrary function of the form

$$
f(\hat{\mathbf{u}})=F_{0}+\mathbf{F}_{2}: \mathbf{q}+\cdots,
$$

it follows from orthogonality and from the identities (B6) that the expansion coefficients $F_{0}$ and $\mathbf{F}_{2}$ can be found from

$$
\begin{gathered}
F_{0}=\frac{1}{4 \pi} \oint d \hat{\mathbf{u}} f(\hat{\mathbf{u}}), \\
\mathbf{F}_{2}=\frac{15}{8 \pi} \oint d \hat{\mathbf{u}} \mathbf{q} f(\hat{\mathbf{u}}),
\end{gathered}
$$

where in the last equation it is used, without loss of generality, that $\mathbf{F}_{2}$ is a traceless and symmetric tensor. There is one term in the expansion (8), when multiplied with $\mathbf{k} \cdot \mathbf{D}(\hat{\mathbf{u}}) \cdot \mathbf{k}$ that is of third order in q. The corresponding zeroth and linear contributions in the orientational order parameter [the ones related to $q_{0}$ and $\mathbf{q}_{2}$ in an expansion of the form (B14)] can be found from (again, summation over repeated indices in implied)

$$
\begin{gathered}
\hat{k}_{k} \hat{k}_{l} \hat{k}_{p} \hat{k}_{q} \oint d \hat{\mathbf{u}} q_{p q} q_{k l} q_{m n} A_{2, m n}=\frac{32 \pi}{315} \hat{\mathbf{k}} \hat{\mathbf{k}}: \mathbf{A}_{2}, \\
\hat{k}_{k} \hat{k}_{l} \hat{k}_{p} \hat{k}_{q} \oint d \hat{\mathbf{u}} q_{i j} q_{p q} q_{k l} q_{m n} A_{2, m n} \\
=\frac{32 \pi}{945} A_{2, i j}-\frac{32 \pi}{945} \delta_{i j} \hat{\mathbf{k}} \hat{\mathbf{k}}: \mathbf{A}_{2}+\frac{32 \pi}{315} \hat{k}_{i} \hat{k} \hat{k}_{j} \hat{\mathbf{k}} \mathbf{k}: \mathbf{A}_{2} .
\end{gathered}
$$

The derivation of these identities relies on a considerable bookkeeping of indices, using the identities (B7) and (B8). Bilinear terms in $\mathbf{q}$ are more conveniently handled by means of the identities

$$
\hat{\mathbf{k}} \hat{\mathbf{k}}: \mathbf{q q}: \hat{\mathbf{k}} \hat{\mathbf{k}}=\hat{\mathbf{k}} \hat{\mathbf{k}}: \mathbf{q}_{4}: \hat{\mathbf{k}} \hat{\mathbf{k}}+\frac{4}{21} \hat{\mathbf{k}} \hat{\mathbf{k}}: \mathbf{q}+\frac{4}{45},
$$




$$
\begin{aligned}
\hat{\mathbf{k}} \hat{\mathbf{k}}: \mathbf{q q}: \mathbf{A}_{2}= & \hat{\mathbf{k}} \hat{\mathbf{k}}: \mathbf{q}_{4}: \mathbf{A}_{2}+\mathbf{q}:\left\{-\frac{4}{21} \mathbf{A}_{2}+\frac{4}{7} \mathbf{A}_{2} \cdot \hat{\mathbf{k}} \hat{\mathbf{k}}\right\} \\
& +\frac{2}{15} \mathbf{A}_{2}: \hat{\mathbf{k}} \hat{\mathbf{k}},
\end{aligned}
$$

$$
\begin{aligned}
\hat{\mathbf{k}} \hat{\mathbf{k}}: \mathbf{q q}:\left(\mathbf{A}_{2} \cdot \hat{\mathbf{k}} \hat{\mathbf{k}}\right)= & \hat{\mathbf{k}} \hat{\mathbf{k}}: \mathbf{q}_{4}:\left(\mathbf{A}_{2} \cdot \hat{\mathbf{k}} \hat{\mathbf{k}}\right)+\mathbf{q}:\left\{\frac{2}{21} \mathbf{A}_{2} \cdot \hat{\mathbf{k}} \hat{\mathbf{k}}\right. \\
& \left.+\frac{2}{21} \hat{\mathbf{k}} \hat{\mathbf{k}}\left(\mathbf{A}_{2}: \hat{\mathbf{k}} \hat{\mathbf{k}}\right)\right\}+\frac{4}{45}\left(\mathbf{A}_{2}: \hat{\mathbf{k}} \hat{\mathbf{k}}\right) .
\end{aligned}
$$

Multiplying Eq. (8) with

$$
\mathbf{k} \cdot[\hat{\mathbf{u}} \hat{\mathbf{u}}+\hat{\mathbf{I}}] \cdot \mathbf{k}=k^{2}\left[\hat{\mathbf{k}} \hat{\mathbf{k}}: \mathbf{q}+\frac{4}{3}\right]
$$

straightforwardly leads to (here $\odot$ stand for a fourfold contraction)

$$
\begin{aligned}
&-\mathbf{k} \cdot \mathbf{D}(\hat{\mathbf{u}}) \cdot \mathbf{k} \oint d \hat{\mathbf{u}}^{\prime} W\left(\mathbf{k}, \hat{\mathbf{u}}, \hat{\mathbf{u}}^{\prime}\right) \delta \rho\left(\mathbf{k}, \hat{\mathbf{u}}^{\prime}, t\right) \\
&=- 2 \frac{L}{D} \varphi \bar{D} k^{2}\left\{F^{2} A_{0}+\frac{13}{96} G \mathbf{A}_{2}: \hat{\mathbf{k}} \hat{\mathbf{k}}+\mathbf{q}:\left[\left(-\frac{1}{8} F^{2}\right.\right.\right. \\
&\left.\left.+\frac{1}{48} G\right) \mathbf{A}_{2}+\frac{7}{8} G A_{0} \hat{\mathbf{k}} \hat{\mathbf{k}}-\frac{1}{16} G \mathbf{A}_{2} \cdot \hat{\mathbf{k}} \hat{\mathbf{k}}\right] \\
&+\frac{21}{32} G A_{0} \hat{\mathbf{k}} \hat{\mathbf{k}}: \mathbf{q q}: \hat{\mathbf{k}} \hat{\mathbf{k}}-\left(\frac{3}{32} F^{2}+\frac{3}{128} G\right) \hat{\mathbf{k}} \hat{\mathbf{k}}: \mathbf{q q}: \mathbf{A}_{2} \\
&\left.-\frac{3}{64} G \hat{\mathbf{k}} \hat{\mathbf{k}}: \mathbf{q q}:\left(\mathbf{A}_{2} \cdot \hat{\mathbf{k}} \hat{\mathbf{k}}\right)-\frac{27}{256} G \hat{\mathbf{k}} \hat{\mathbf{k}} \hat{\mathbf{k}} \hat{\mathbf{k}} \odot \mathbf{q q q}: \mathbf{A}_{2}\right\} .
\end{aligned}
$$

The zeroth and linear order terms in $\mathbf{q}$ arising from the bilinear terms in $\mathbf{q}$ in the above equation can be found by substitution of the identities (B17), while these contributions arising from the last term $\sim \mathbf{q q q}$ are more conveniently found from the relation (B15) and the identity (B16). This leads, up to linear order in the orientational order parameter, to

$$
\begin{aligned}
-\mathbf{k} & \cdot \mathbf{D}(\hat{\mathbf{u}}) \cdot \mathbf{k} \oint d \hat{\mathbf{u}}^{\prime} W\left(\mathbf{k}, \hat{\mathbf{u}}, \hat{\mathbf{u}}^{\prime}\right) \delta \rho\left(\mathbf{k}, \hat{\mathbf{u}}^{\prime}, t\right) \\
= & -2 \frac{L}{D} \varphi \bar{D} k^{2}\left\{\left(F^{2}+\frac{7}{120} G\right) A_{0}-\left(\frac{1}{80} F^{2}-\frac{47}{420} G\right) \mathbf{A}_{2}: \hat{\mathbf{k}} \hat{\mathbf{k}}\right. \\
& +\mathbf{q}:\left[\left(\frac{3}{4} F^{2}+G\right) A_{0} \hat{\mathbf{k}} \hat{\mathbf{k}}-\left(\frac{3}{28} F^{2}-\frac{17}{448} G\right) \mathbf{A}_{2}-\left(\frac{3}{56} F^{2}\right.\right. \\
& \left.\left.+\frac{31}{224} G\right) \mathbf{A}_{2} \cdot \hat{\mathbf{k}} \hat{\mathbf{k}}+\frac{69}{896} G \hat{\mathbf{k}} \hat{\mathbf{k}}\left(\mathbf{A}_{2}: \hat{\mathbf{k}} \hat{\mathbf{k}}\right)\right] \\
& \left.+\mathcal{O}\left((k L)^{4}, \lambda^{2}\right)\right\} .
\end{aligned}
$$

Here, terms $\sim \mathbf{q}_{4}$ are omitted, being of second order in the orientational order parameter $\lambda$.
The free translational contribution to the equation of motion is similarly found to be equal to

$$
\begin{aligned}
&-\mathbf{k} \cdot \mathbf{D}(\hat{\mathbf{u}}) \cdot \mathbf{k} \delta \rho(\mathbf{k}, \hat{\mathbf{u}}, t) \\
&=-\bar{D} k^{2}\left\{A_{0}+\frac{1}{10} \mathbf{\mathbf { A } _ { 2 }}: \hat{\mathbf{k}} \hat{\mathbf{k}}+\mathbf{q}:\left[\frac{6}{7} \mathbf{A}_{2}+\frac{3}{4} A_{0} \hat{\mathbf{k}} \hat{\mathbf{k}}\right.\right. \\
&\left.\left.+\frac{3}{7} \mathbf{A}_{2} \cdot \hat{\mathbf{k}} \hat{\mathbf{k}}\right]+\mathcal{O}\left((k L)^{2}, \lambda^{2}\right)\right\} .
\end{aligned}
$$

Next, using that $\left(\partial_{k}\right.$ is the differentiation with respect to the $k$ th component $\hat{u}_{k}$ of $\hat{\mathbf{u}}$ and, as before, summation over repeated indices is understood)

$$
[\hat{\mathcal{R}}(\cdots)]_{i}=\epsilon_{i j k} \hat{u}_{j} \partial_{k}, \quad \text { and } \epsilon_{i j k} \epsilon_{r s k}=\delta_{i r} \delta_{j s}-\delta_{i s} \delta_{j r},
$$

where $\epsilon_{i j k}$ is the Levi-Cevita tensor, it is easily shown that

$$
\begin{gathered}
\hat{\mathcal{R}}^{2} \hat{u}_{i} \hat{u}_{j}=2\left[\delta_{i j}-3 \hat{u}_{i} \hat{u}_{j}\right]=-6 q_{i j}, \\
\hat{\mathcal{R}}^{2} \hat{u}_{i} \hat{u}_{j} \hat{u}_{k} \hat{u}_{l}=-20 \hat{u}_{i} \hat{u}_{j} \hat{u}_{k} \hat{u}_{l}+2\left[\hat{u}_{i} \hat{u}_{k} \delta_{j l}+\hat{u}_{i} \hat{u}_{l} \delta_{j k}+\hat{u}_{j} \hat{u}_{k} \delta_{i l}\right. \\
\left.+\hat{u}_{j} \hat{u}_{l} \delta_{i k}+\hat{u}_{i} \hat{u}_{j} \delta_{k l}+\hat{u}_{k} \hat{u}_{l} \delta_{i j}\right] .
\end{gathered}
$$

From the definition of $\mathbf{q}_{4}$ in Eq. (B9) it is readily found from the above identities that

$$
\hat{\mathbf{k}} \hat{\mathbf{k}}: \hat{\mathcal{R}}^{2} \mathbf{q q}: \mathbf{A}_{2}=\hat{\mathbf{k}} \hat{\mathbf{k}}: \mathbf{q}_{4}: \mathbf{A}_{2}-20 \mathbf{q}:\left\{\frac{5}{84} \mathbf{A}_{2}+\frac{6}{35} \mathbf{A}_{2} \cdot \hat{\mathbf{k}} \hat{\mathbf{k}}\right\} .
$$

It thus follows from Eq. (8) that, again up to order $k^{2}$ and linear order in orientational order parameter, that

$$
\begin{aligned}
D_{r} \hat{\mathcal{R}}^{2} \oint d \hat{\mathbf{u}}^{\prime} W\left(\mathbf{k}, \hat{\mathbf{u}}, \hat{\mathbf{u}}^{\prime}\right) \delta \rho\left(\mathbf{k}, \hat{\mathbf{u}}^{\prime}, t\right) \\
\quad=2 \frac{L}{D} \varphi D_{r} \mathbf{q}:\left[\left(\frac{3}{4} F^{2}+\frac{19}{448} G\right) \mathbf{A}_{2}+\frac{6}{7} G \mathbf{A}_{2} \cdot \hat{\mathbf{k}} \hat{\mathbf{k}}\right] .
\end{aligned}
$$

Similarly, the free rotational contribution to the equation of motion is found to be equal to

$$
D_{r} \hat{\mathcal{R}}^{2} \delta \rho(\mathbf{k}, \hat{\mathbf{u}}, t)=-6 D_{r} \mathbf{q}: \mathbf{A}_{2} .
$$

Collecting terms in Eqs. (B20), (B21), (B25), and (B26), and equating the coefficients of $q_{0}$ and $\mathbf{q}_{2}$ (in symmetric and traceless form), leads to the equations of motion (30) and (31).

\section{APPENDIX C: EXACT EXPRESSIONS FOR DIFFUSION COEFFICIENTS}

The expressions in Eq. (37) for the diffusion coefficients are obtained from the simplified equations of motion (33) and (34). The analogous expressions as obtained from the equations of motion (30) and (31) read

$$
D_{11}=\bar{D}\left\{1+2 \frac{L}{D} \varphi\left[1-\frac{29}{960}(k L)^{2}\right]\right\},
$$




$$
\begin{gathered}
D_{12}=\frac{1}{10} \bar{D}\left\{1-\frac{1}{4} \frac{L}{D} \varphi\left[1+\frac{29}{84}(k L)^{2}\right]\right\}, \\
D_{21}=\frac{1}{2} \bar{D}\left\{1+2 \frac{L}{D} \varphi\left[1-\frac{1}{12}(k L)^{2}\right]\right\}, \\
D_{22}=6 D_{r}\left\{1-\frac{1}{4} \frac{L}{D} \varphi\left[1-\frac{499}{8064}(k L)^{2}\right]\right\} \\
+\frac{8}{7} \bar{D} k^{2}\left\{1-\frac{1}{4} \frac{L}{D} \varphi\left[1-\frac{53071}{1334786}(k L)^{2}\right]\right\} .
\end{gathered}
$$

The eigenvalues calculated from these expressions are essentially equal to those related to the simplified version in Eq. (37) as plotted in Fig. 3.

\section{APPENDIX D: SMALL ANGLE LIGHT SCATTERING BY DEMIXING SUSPENSIONS OF RODS}

When the refractive index difference along and perpendicular to the long axis of the rod is small as compared to the average refractive index difference with the solvent, the scattered intensity is equal to

$$
I \sim\left\langle\sum_{i, j=1}^{N} \exp \left[i \mathbf{k} \cdot\left(\mathbf{r}_{i}-\mathbf{r}_{j}\right)\right] B\left(\mathbf{k}, \hat{\mathbf{u}}_{i}\right) B\left(-\mathbf{k}, \hat{\mathbf{u}}_{j}\right)\right\rangle
$$

where the brackets $\langle\cdots\rangle$ denote ensemble averaging, while

$$
B(\mathbf{k}, \hat{\mathbf{u}})=\int_{V^{(0)}(\mathbf{u})} d \mathbf{r} \exp \{i \mathbf{k} \cdot \mathbf{r}\} .
$$

Here, $V^{(0)}(\mathbf{u})$ is the volume occupied by a rod with orientation $\hat{\mathbf{u}}$ and with its center at the origin [as indicated by the superscript (0)]. When $k D<1$ the "scattering amplitude" $B$ is simply equal to

$$
B(\mathbf{k}, \hat{\mathbf{u}})=\frac{\pi}{4} D^{2} \int_{-(1 / 2) L}^{(1 / 2) L} d l \exp \left\{i \mathbf{k} \cdot \hat{\mathbf{u}}_{j} l\right\}=\frac{\pi}{4} D^{2} L j_{0}\left(\frac{1}{2} L \mathbf{k} \cdot \hat{\mathbf{u}}_{j}\right),
$$

where, as before, $j_{0}(x)=\sin \{x\} / x$. Hence

$$
I \sim\left\langle\sum_{i, j=1}^{N} \exp \left[i \mathbf{k} \cdot\left(\mathbf{r}_{i}-\mathbf{r}_{j}\right)\right] j_{0}\left(\frac{1}{2} L \mathbf{k} \cdot \hat{\mathbf{u}}_{1}\right) j_{0}\left(\frac{1}{2} L \mathbf{k} \cdot \hat{\mathbf{u}}_{2}\right)\right\rangle .
$$

Using the definition (7) of the pair-correlation function and introducing the total-correlation function $h=g+1$, the scattered intensity can be written as

$$
\begin{aligned}
I & \sim \int d \mathbf{r}_{1} \int d \mathbf{r}_{2} \oint d \hat{\mathbf{u}}_{1} \oint d \hat{\mathbf{u}}_{2} P_{2}\left(\mathbf{r}_{1}, \mathbf{r}_{2}, \hat{\mathbf{u}}_{1}, \hat{\mathbf{u}}_{2}, t\right) \exp \left[i \mathbf{k} \cdot\left(\mathbf{r}_{1}-\mathbf{r}_{2}\right)\right] j_{0}\left(\frac{1}{2} L \mathbf{k} \cdot \hat{\mathbf{u}}_{1}\right) j_{0}\left(\frac{1}{2} L \mathbf{k} \cdot \hat{\mathbf{u}}_{2}\right) \\
= & \int d \mathbf{r}_{1} \int d \mathbf{r}_{2} \oint d \hat{\mathbf{u}}_{1} \oint d \hat{\mathbf{u}}_{2} \rho\left(\mathbf{r}_{1}, \hat{\mathbf{u}}_{1}, t\right) \rho\left(\mathbf{r}_{2}, \hat{\mathbf{u}}_{2}, t\right) h\left(\mathbf{r}_{1}, \mathbf{r}_{2}, \hat{\mathbf{u}}_{1}, \hat{\mathbf{u}}_{2}, t\right) \exp \left[i \mathbf{k} \cdot\left(\mathbf{r}_{1}-\mathbf{r}_{2}\right)\right] j_{0}\left(\frac{1}{2} L \mathbf{k} \cdot \hat{\mathbf{u}}_{1}\right) j_{0}\left(\frac{1}{2} L \mathbf{k} \cdot \hat{\mathbf{u}}_{2}\right) \\
& +\int d \mathbf{r}_{1} \int d \mathbf{r}_{2} \oint d \hat{\mathbf{u}}_{1} \oint d \hat{\mathbf{u}}_{2} \rho\left(\mathbf{r}_{1}, \hat{\mathbf{u}}_{1}, t\right) \rho\left(\mathbf{r}_{2}, \hat{\mathbf{u}}_{2}, t\right) \exp \left[i \mathbf{k} \cdot\left(\mathbf{r}_{1}-\mathbf{r}_{2}\right)\right] j_{0}\left(\frac{1}{2} L \mathbf{k} \cdot \hat{\mathbf{u}}_{1}\right) j_{0}\left(\frac{1}{2} L \mathbf{k} \cdot \hat{\mathbf{u}}_{2}\right)
\end{aligned}
$$

assuming identical rods. By definition, $h=0$ for distances $\left|\mathbf{r}_{1}-\mathbf{r}_{2}\right|>L$. The wave-vector dependence of the first term on the right-hand side of Eq. (D5) is therefore weak for $k L<1$. Furthermore, the range of integration with respect to the distance between rods is of the order $L$ in the first term, while there is no natural bound for this integration range for the last term. The first term is therefore small in comparison to the last term, provided that inhomogeneities are developed to some extent. Hence

$$
\begin{aligned}
I & \sim \oint d \hat{\mathbf{u}}_{1} \oint d \hat{\mathbf{u}}_{2} \rho\left(\mathbf{k}, \hat{\mathbf{u}}_{1}, t\right) \rho\left(\mathbf{k}, \hat{\mathbf{u}}_{2}, t\right) j_{0}\left(\frac{1}{2} L \mathbf{k} \cdot \hat{\mathbf{u}}_{1}\right) j_{0}\left(\frac{1}{2} L \mathbf{k} \cdot \hat{\mathbf{u}}_{2}\right) \\
& =\left[\oint d \hat{\mathbf{u}} \rho(\mathbf{k}, \hat{\mathbf{u}}, t) j_{0}\left(\frac{1}{2} L \mathbf{k} \cdot \hat{\mathbf{u}}\right)\right]^{2} .
\end{aligned}
$$

Note that when the system is homogeneous, $\rho(\mathbf{k}, \hat{\mathbf{u}}, t)$ would be a delta function of $\mathbf{k}$. In that case this contribution is zero for nonzero scattering vectors, and the only remaining contribution to the scattered intensity is the first term on the right-hand side of Eq. (D5). Since $A_{0} \sim k^{2}$, being equal to the number density, the function $j_{0}$ must be expanded up to $k^{4}$ in order to obtain the integral in Eq. (D6) up to order $k^{4}$. Using that $j_{0}(x)=1-1 / 6 x^{2}+1 / 120 x^{4}+\cdots$, substitution of the representation (20) and using the identities (B6) and (B7) leads to the expression (46) for the scattered intensity. 
[1] J. W. Cahn, and J. E. Hilliard, J. Chem. Phys. 28, 258 (1958); 31, 688 (1959).

[2] J. W. Cahn, J. Chem. Phys. 42, 93 (1965).

[3] J. S. Langer, M. Bar-on, and H. D. Miller, Phys. Rev. A 11, 1417 (1975).

[4] K. Kawasaki, and T. Ohta, Prog. Theor. Phys. 59, 362 (1978); 59, 1406 (1978).

[5] K. Binder, Colloid Polym. Sci. 265, 273 (1987).

[6] G. F. Mazenko, Phys. Rev. B 42, 4487 (1990).

[7] A. Sariban, and K. Binder, Macromolecules 24, 578 (1991).

[8] P. Fratzl, J. L. Lebowitz, O. Penrose, and J. Amar, Phys. Rev. B 44, 4794 (1991).

[9] A. Shinozaki, and Y. Oono, Phys. Rev. Lett. 66, 173 (1991).

[10] F. J. Alexander, S. Chen, and D. W. Grunau, Phys. Rev. B 48, R634 (1993).

[11] T. Koga, and K. Kawasaki, Physica A 196, 389 (1993).

[12] J. K. G. Dhont, J. Chem. Phys. 105, 5112 (1996).

[13] J. K. G. Dhont, Prog. Colloid Polym. Sci. 104, 66 (1997).

[14] J. K. G. Dhont, in Dynamics: Models and Kinetic Methods for Non-equilibrium Many Body Systems, edited by J. Karkheck (Kluwer Academic Publishers, Amsterdam, 2000), p. 73.

[15] K. Binder, and D. Stauffer, Phys. Rev. Lett. 33, 1006 (1974).

[16] E. D. Siggia, Phys. Rev. A 20, 595 (1979).

[17] K. Binder, Rep. Prog. Phys. 50, 783 (1987).

[18] W. I. Goldburg, in Scattering Techniqies Applied to Supramolecular and Nonequilibrium Systems, edited by S. H. Chen et al. (Plenum Press, New York, 1981), p. 383.

[19] J. D. Gunton, M. San Miquel, and P. S. Sahni, in Phase Transitions and Critical Phenomena, edited by C. Domb and J. L. Lebowitz (Academic Press, New York, 1983), Vol. 8, p. 267.

[20] S. W. Koch, in Dynamics of First-Order Phase Transitions in Equilibrium and Nonequilibrium Systems, edited by H. Araki et al., Lecture Notes in Physics (Springer-Verlag, Berlin, 1984).

[21] K. Binder, and D. W. Heermann, in Scaling Phenomena in Disordered Systems, edited by R. Pynn, and A. Skjeltorp (Plenum Press, New York, 1985), p. 207.
[22] H. Furukawa, Adv. Phys. 34, 703 (1985).

[23] P. Guyot, J. P. Simon, J. Chim. Phys. Phys.-Chim. Biol. 83, 703 (1986).

[24] D. G. A. L. Aarts, R. P. A. Dullens, and H. N. W. Lekkerkerker, New J. Phys. 7, 40 (2005).

[25] T. Shimada, M. Doi, and K. Okano, J. Chem. Phys. 88, 7181 (1988).

[26] J. W. Winters, Th. Odijk, and P. van der Schoot, Phys. Rev. E 63, 011501 (2000).

[27] J. Tang, and S. Fraden, Phys. Rev. Lett. 71, 3509 (1993).

[28] M. R. Stukan, V. A. Ivanov, M. Müller, W. Paul, and K. Binder, e-Polymers 062 (2003) [http://www.e-polymers.org, ISSN 1618-7229].

[29] M. Doi, T. Shimada, and K. Okano, J. Chem. Phys. 88, 4070 (1988).

[30] T. Maeda, Macromolecules 22, 1881 (1989).

[31] M. Doi, and S. F. Edwards, The Theory of Polymer Dynamics (Clarendon Press, Oxford, 1986).

[32] J. K. G. Dhont, An Introduction to Dynamics of Colloids (Elsevier, Amsterdam, 1996).

[33] L. Onsager, Chem. Rev. (Washington, D.C.) 13, 73 (1933).

[34] L. Onsager, Phys. Rev. 62, 558 (1942).

[35] L. Onsager, Ann. N.Y. Acad. Sci. 51, 627 (1949).

[36] G. J. Vroege, and H. N. W. Lekkerkerker, Rep. Prog. Phys. 55, 1241 (1992).

[37] J. K. G. Dhont, and W. J. Briels, in Soft Condensed Matter, edited by G. Gompper and M. Schick (Wiley-VCH, in press).

[38] Y. Tao, W. K. den Otter, J. T. Padding, J. K. G. Dhont, and W. J. Briels, (unpublished).

[39] R. F. Kayser, and H. J. Raveché, Phys. Rev. A 17, 2067 (1978).

[40] J. K. G. Dhont, and W. J. Briels, Colloids Surf., A 213, 131 (2003).

[41] K. Kang, P. Lettinga, and D. Derks (private communication).

[42] A. Matsuyama, R. M. L. Evans, and M. E. Cates, Phys. Rev. E 61, 2977 (2000). 